\title{
Paraxial mesoderm organoids model development of human somites
}

2 Christoph Budjan ${ }^{1,2}$, Shichen Liu ${ }^{1,2}$, Adrian Ranga ${ }^{3}$, Senjuti Gayen ${ }^{1,2}$, Olivier Pourquie ${ }^{4,5}$, 3 Sahand Hormoz ${ }^{1,2,6}$

4 Affiliations

5 'Department of Systems Biology, Harvard Medical School, Boston, MA, USA

$6{ }^{2}$ Department of Data Sciences, Dana-Farber Cancer Institute, Boston, MA, USA

7 ' ${ }^{3}$ Laboratory of Bioengineering and Morphogenesis, Biomechanics Section, Department of

8 Mechanical Engineering, KU Leuven, Leuven, Belgium

$9{ }^{4}$ Department of Genetics, Harvard Medical School, Boston, MA, USA

$10{ }^{5}$ Department of Pathology, Brigham and Women's Hospital, Boston, MA, USA

$11{ }^{6}$ Broad Institute of MIT and Harvard, Cambridge, MA, USA

12 Correspondence: sahand hormoz@hms.harvard.edu

\section{Abstract}

14 During the development of the vertebrate embryo, segmented structures called somites are periodically formed from the presomitic mesoderm (PSM), and give rise to the vertebral column. While somite formation has been studied in several animal models, it

17 is less clear how well this process is conserved in humans. Recent progress has made

18 it possible to study aspects of human paraxial mesoderm development such as the human segmentation clock in vitro using human pluripotent stem cells (hPSCs), however, somite formation has not been observed in these monolayer cultures. Here, we describe the generation of human paraxial mesoderm (PM) organoids from hPSCs (termed Somitoids), which recapitulate the molecular, morphological and functional features of paraxial mesoderm development, including formation of somite-like

24 structures in vitro. Using a quantitative image-based screen, we identify critical parameters such as initial cell number and signaling modulations that reproducibly yielded formation of somite-like structures in our organoid system. In addition, using

27 single-cell RNA sequencing and 3D imaging, we show that PM organoids both transcriptionally and morphologically resemble their in vivo counterparts and can be differentiated into somite derivatives. Our organoid system is reproducible and scalable,

30 allowing for the systematic and quantitative analysis of human spinal cord development 31 and disease in vitro. 


\section{Introduction}

33 Paraxial mesoderm (PM) development involves the formation of embryonic segments

34 called somites, which are produced sequentially from the presomitic mesoderm (PSM) 35 and arranged periodically along the anterior-posterior (AP) axis of the vertebrate embryo. Somites give rise and contribute to a variety of tissues including skeletal muscle, dermis, cartilage and bone (Chal and Pourquié, 2017). Somite formation is controlled by a conserved molecular oscillator, the segmentation clock (Dequeant et al., 2006; Hubaud and Pourquié, 2014; Oates et al., 2012). Previous efforts have focused on how this oscillator controls somite formation using a variety of model systems such as mouse, zebrafish, and chick, because of ethical and technical limitations of culturing human embryos. Recently, researchers were able to recapitulate paraxial mesoderm development using human and mouse pluripotent stem cells cultured as 2D monolayers (Chu et al., 2019; Diaz-Cuadros et al., 2020; Matsuda et al., 2020). These cells undergo species-characteristic oscillations similar to their in vivo counterparts. However, final stages of somite development and vertebra formation was not observed in currently published human cell culture systems (Palla and Blau, 2020), suggesting that certain aspects of in vivo development are not recapitulated in these 2D systems. We reasoned that a 3D cell culture system may exhibit all the stages of PM development including morphogenetic processes associated with somite formation.

Here, we describe an organoid system derived from human iPS cells (hiPSCs) called Somitoids, which faithfully recapitulates functional, morphological and molecular

53 features of paraxial mesoderm development, including formation of somite-like 54 structures in vitro. To identify the culture conditions that reproducibly yielded formation of somite-like structures, we developed a quantitative image-based screening platform 56 for individual organoids. Our screening approach identified the optimal parameter 57 values for the culture conditions such as the initial cell number and the concentration of 58 the chemical modulators. We show using single-cell RNA-sequencing (scRNA-seq), 59 immunofluorescence, and qRT-PCR that Somitoids resemble their in vivo counterparts 60 both transcriptionally and morphologically and can be differentiated into somite derivatives such as sclerotome and dermomyotome in vitro. 
Our Somitoid system is reproducible and scalable, allowing for systematic and quantitative analysis of paraxial mesoderm development and somite formation to study

64 human spinal cord development and disease in a dish. Finally, our approach can be used to systematically screen organoid cultures for desired phenotypes and 66 reproducibility.

\section{Results}

Recently, protocols have been developed to differentiate mouse or human pluripotent stem cells towards paraxial mesoderm using a combination of the WNT agonist CHIR and BMP inhibitor LDN (Chal et al., 2015; Diaz-Cuadros et al., 2020). To adapt the protocol for a 3D model of human somitogenesis, we first optimized the initial conditions of our cultures by generating pluripotent spheroids of defined cell numbers. hiPS cells were allowed to aggregate for 24 hours as suspension cultures in pluripotency media (mTeSR1) in the presence of ROCK inhibitor and Polyvinyl alcohol (PVA) to promote aggregation (Fig 1A). These pluripotent spheroids resemble cavity-stage epiblast embryos as previously described for kidney organoid cultures (Freedman et al., 2015)(Fig 1B and Fig 1-Supplemental Fig 1A). Next, spheroids were cultured in media containing CHIR and LDN (CL), similar as done for the monolayer cultures (Chal et al., 2015; Diaz-Cuadros et al., 2020) but with CHIR at a higher concentration (10 $\mu \mathrm{M})$. After 24 hours in CL media, epiblast-stage cells transition to a neuromesodermal (NMp) or primitive streak cell fate, characterized by co-expression of T/BRA and SOX2 (Tzouanacou et al., 2009)(Fig 1B). By 48 hours, cells rapidly downregulate SOX2 and express PSM markers, including TBX6 and MSGN1 (Fig 1B). This PSM state persists from day 2 to day 4 and is also characterized by the expression of segmentation clock genes such as HES7 (Fig 1C). On day 5, organoids showed expression of marker genes associated with somite fate as characterized by qPCR (Fig 1C, Fig 1Supplemental Fig 1B). Taken together, the order of activation of marker genes in the paraxial mesoderm organoids followed the expected stages of differentiation observed during PM development.

We observed that following the above protocol resulted in a heterogeneous 92 activation of somite marker genes across cells within the same organoid and across 
93 different replicates, as well as a low number of somite-like structures (Figure 194 Supplemental Fig 2). To improve reproducibility, we set out to screen for the optimal initial cell number, the concentration of the signaling factors and the timing of their additions during culture. For our primary screen, we compared organoids with an initial cell number of 500 and 1000 as well as combinations of modulators of candidate signaling pathways that have previously been involved in paraxial mesoderm development and somite formation, including FGF, WNT, BMP and TGF- $\beta$ (Aulehla et al., 2008; Chal et al., 2015; Hubaud and Pourquié, 2014; Tonegawa et al., 1997; Xi et al., 2017). We primarily focused on the FGF and WNT signaling pathways since their critical role during somitogenesis is well established both in vivo (Aulehla et al., 2008, 2003; Delfini et al., 2005; Dubrulle et al., 2001; Dunty et al., 2008; Greco et al., 1996; Yamaguchi et al., 1999) and in vitro (Chal et al., 2015; Sakurai et al., 2012; Tan et al., 2013). Furthermore, dual inhibition of FGF and WNT signaling has been used with some success to generate paraxial mesoderm derivatives in vitro (Loh et al., 2016; Matsuda et al., 2020). Finally, the BMP and TGF- $\beta$ signaling pathways have been shown in vitro and in vivo to have a role in human somitogenesis (Loh et al., 2016; Xi et al., 2017).

PSM-stage organoids on day 3 were treated with signaling modulators for 24 and 48 hours, and somite fate and morphogenesis was assessed using PAX3, a somite fate marker, and F-ACTIN, a structural marker of somite formation (Fig 2A). We chose

113 day 3 as a starting point for our systematic screen because PSM marker gene expression was more uniform compared to day 2 organoids (Fig 1B), and day 4

115 organoids are not significantly different from day 3 organoids based on a previous study 116 and our own immunostaining and qPCR data (Figure 1B,C and Diaz-Cuadros et al., 117 2020). To quantitatively compare conditions, we developed an image analysis pipeline 118 to determine organoid diameter and normalized average PAX3 expression intensity per 119 organoid in an automated manner (Fig 2C,D and Fig 2-Supplemental Fig 2B). We 120 analyzed three organoids per condition. Strikingly, all organoids that were treated with 121 any combination of FGF or WNT inhibitor reproducibly expressed PAX3 within 24 hours 122 of treatment (Fig 2B,D). However, organoids that were initiated from 1000 cells 123 displayed a higher fraction of PAX3-negative cells compared with organoids initiated 
124 from 500 cells, even though the average PAX3 expression levels across the entire 125 organoid was comparable (Figure 2D and Figure 2-Supplemental Fig 2A). Additional 126 staining for SOX2, a neural marker, showed that PAX3-negative cells expressed SOX2, 127 suggesting that our paraxial mesoderm organoids derive from neuro-mesodermal 128 progenitors (Fig 1B and Fig 2-Supplemental Fig 2A). In addition, staining for F-ACTIN, 129 together with nuclear expression of PAX3, more consistently revealed somite-like 130 structures (radial arrangement of PAX3+ columnar cells with expression of apical F131 ACTIN in the central cavity) in organoids made from 500 cells compared with organoids 132 made from 1000 cells (Figure 2B and Figure 2-Supplemental Fig 3A,B). Taken together, 133 based on these observations, we used 500 cells as the initial cell number going forward. 134 Organoids that were treated for 48 hours with signaling pathway modulators 135 showed an overall decrease of PAX3 expression compared with organoids treated for only 24 hours across replicates, indicating that prolonged signaling manipulation does 137 not improve the somite phenotype (Fig 2D). Additionally, organoids initiated from 500 138 cells and then treated for $48 \mathrm{hrs}$ had a smaller diameter compared with organoids of the same initial cell number that were treated for only 24 hours (Fig 2C). This suggests that 140 long-term inhibition of WNT and/or FGF, known mitogenic signaling pathways, has detrimental effects on proliferation or cell survival. These results indicate that treatment of PSM-stage organoids with pathway modulators for 24 hours is sufficient to induce somite fate.

Next, we set out to optimize the culture conditions to increase the number of somite-like structures in addition to the expression levels of the somite marker genes.

146 We looked for morphological hallmarks of somite formation, specifically the formation of 147 rosette-like structures consisting of radially arranged bottle-shaped PAX3+ epithelial 148 cells with their NCAD+ apical surface facing a central cavity (Figure 3-Supplemental Fig 149 1 A,B and Figure 3-Video 1).

150 We used 500-cell spheroids as an initial starting point and compared two 151 different inhibitor doses for FGF and WNT in addition to the other pathway modulators 152 applied to PSM-stage organoids on day 3. We characterized the organoids after treating 153 them for 24 hours, 48 hours, and 24 hours followed by culture in basal media without 154 any added factor for an additional 24 hours ( 5 organoids per condition, Fig 3A). In 
155 addition to quantifying PAX3 levels (Fig 3D, Fig 3-Supplemental Fig 2B), we also 156 counted the number of somite-like structures per organoid (Fig 3B, Fig 3E, Fig 3157 Supplemental Fig 2A,B; see Methods section for a description of the scoring criteria). 158 Comparing PAX3 expression levels in our treated organoids, we observed that somite 159 fate can be broadly induced across a range of treatment regimes, concentrations and 160 types of WNT and/or FGF inhibitors (Fig 3C,D and Fig 3-Supplemental Fig 2C). 161 However, the number of somite-like structures is not necessarily correlated with 162 average PAX3 expression levels. For example, the numbers of somite-like structures in 163 several conditions (FGFRi ${ }^{\mathrm{hi}} / \mathrm{PD} 173, \mathrm{WNTi}^{\mathrm{hi}} / \mathrm{C} 59, \mathrm{WNT}^{\mathrm{hi}} / \mathrm{C} 59+\mathrm{FGFRi}{ }^{\mathrm{lo}}$, WNTi ${ }^{\mathrm{hi}} / \mathrm{XAV}$ ) 164 were lower in organoids treated for 24 hours followed by culture in basal media for 24 165 hours compared with organoids that were treated with the same inhibitors for 48 hours, 166 even though they exhibited higher PAX3 expression levels on average (Fig 3D,E and Fig 3-Supplemental Fig 2A). This suggests that marker gene expression alone may not be the best predictor when screening for morphologically complex phenotypes such as somite formation.

Surprisingly, organoids which were cultured for an additional 24 hours (day 3 to day 4) in FGF, WNT pathway agonist and BMP inhibitor, considered a treatment control, followed by culture in basal media only for another 24 hours, consistently exhibited the highest number of somite-like structures across all organoid replicates (Fig 3E, Figure 3-Supplemental Fig 1A-B, Fig 3-Supplemental Fig 2A-B and Figure 3-Video 1) as well as technical replicates (Figure 3-Supplemental Fig 3A). Additionally, the average PAX3 expression was among the highest of all conditions tested (Fig 3D). This suggests that simply removing FGF/WNT pathway agonists and BMP inhibitor, which maintain cells in a PSM state, is sufficient to reproducibly induce somite fate and morphological formation of somite-like structures (Fig 3-Supplemental Fig 2A, Fig 3-Supplemental Fig $3 \mathrm{~A})$. Computing the variation of the number of somite-like structures across the 5 organoids confirmed that this phenotype was highly reproducible (coefficient of variation = 11.1\%; Fig 3-Supplemental Fig 2B). Finally, our optimized protocol reproducibly yielded efficient induction of somite-like structures in multiple genetically independent hiPS cell lines (Figure 3-Supplemental Fig 3B). Taken together, we determined that initiating the protocol with 500 cells and treating day 3 organoids with $\mathrm{CL}+\mathrm{FGF}$ for 24 
186 hours followed by culture in basal media for an additional 24 hours yields the most 187 robust induction of somite-like structures while minimizing variation between 188 experiments (technical variation) and different cell lines (biological variation). This 189 optimized differentiation protocol was therefore used for all subsequent experiments.

To further characterize the developmental trajectory and transcriptional states of our Somitoid system, we collected 15,558 cells (after post-processing) over the course of the optimized five day differentiation protocol at time-points that capture the key 193 transition steps (day 1 , day 2, day 3 , day 5 ) and performed single-cell RNA sequencing 194 (scRNA-seq; Fig 4A). Multiple organoids were used to obtain the required number of 195 cells at each timepoint (see Methods). We first combined all the cells across the time196 points and clustered them using the Leiden clustering algorithm (Traag et al., 2019). 197 Predominantly, the 4 major clusters corresponded to cells from the 4 different time198 points. Therefore, cells at each time-point have transcriptional states that are different 199 compared with cells from the other time-points. In addition, within each time-point, cells 200 exhibit similar transcriptional profiles as indicated by the uniformity of the expression levels of marker genes across individual cells (Fig 4E, Fig 4-Supplemental Fig 1,2,3).

Cells collected on day 1 exhibited gene expression profiles similar to primitive streak or neuro-mesodermal progenitors, expressing genes such as SOX2, T/BRA, 204 MIXL1 and NODAL (Fig 4C,E, Fig 4-Supplemental Fig 1). Starting on day 2, cells 205 expressed canonical PSM marker genes such as TBX6, MSGN1, WNT3A, RSPO3 and 206 clock genes of the Notch signaling pathway including HES7, LFNG, DLL1 and DLL3 207 (Fig 4C,E; Fig 4-Supplemental Fig 2). Day 5 cells expressed somite-marker genes such 208 as TCF15, PAX3, FOXC2 and MEOX2 (Fig 4C,E; Fig 4-Supplemental Fig 3). 209 Interestingly, a subset of the day 5 cells also expressed somite polarity markers, UNCX 210 and TBX18, which suggests faithful recapitulation of somite patterning in Somitoids (Fig 211 4C; Fig 4-Supplemental Fig 3). Furthermore, two of the sub-clusters ('PSM-to-Somite', 212 'Early Somite'), which are characterized by co-expression of PSM and somite marker 213 genes, were comprised of both day 3 and day 5 cells, indicating that the PSM-to-somite 214 transition is captured in our in vitro system (Fig 4A,B). Interestingly, one somite sub215 cluster ('Late Somite') was enriched for myogenic genes (MYL4/6/7/9, TROPONIN L1) 216 and sclerotome genes (TWIST1, COL1A1, COL11A1, COL7A1, ACTA2), suggesting 
217 that these cells represent more downstream fates of somite-derived cells (Fig 4B; Fig 4218 Supplemental Fig 4A). Finally, we observed the expected sequential activation pattern 219 of the HOX genes in our Somitoid system starting with HOXA1 on day 1, followed by 220 other cervical and thoracic HOX genes on day 2-3, to HOXD9, a lumbosacral HOX 221 gene, in the somite-stage organoids (day 5; Fig 4D and Fig 4-Supplemental Fig 4B). 222 Taken together, our scRNA-seq analyses show that our Somitoid system faithfully 223 recapitulates the gene regulatory programs of human paraxial mesoderm development 224 and generates mature somite-like cells, which express the full repertoire of known 225 marker genes. Furthermore, we did not detect cells of different developmental origins, 226 suggesting that we are generating homogeneous organoids containing only paraxial 227 mesoderm derivatives.

228 We next assessed whether our in vitro derived somite-like structures show 229 similar spatial organization and size distribution to their in vivo counterparts. To 230 independently confirm some of the somite marker genes that we identified in our 231 scRNA-seq data set, we measured expression levels of TCF15/PARAXIS, PAX3, and 232 F-ACTIN in day 5 Somitoids using whole-mount immunostaining (Fig 5A). Day 5 cells 233 co-expressed both somite marker genes TCF15 and PAX3 throughout the Somitoid and 234 somite-like structures displayed apical localization of F-ACTIN. To determine whether in 235 vitro-derived somite-like structures were similar in size to human embryonic segments, 236 we compared them with Carnegie stage 9-11 early human somites (Fig 5B; see 237 Methods section for a description of how somite sizes were quantified). Organoid238 derived somite-like structures were similar in size (median area $=8892 \mu \mathrm{m}^{2}$, 239 interquartile range/IQR $=7698-10682 \mu \mathrm{m}^{2}$ ) to Carnegie stage 11 somites (median area $240=9681 \mu \mathrm{m}^{2}, \mathrm{IQR}=8262-11493 \mu \mathrm{m}^{2}$ ) but larger than earlier-stage human somites 241 (Carnegie stage 9 somites, median area $=4399 \mu \mathrm{m}^{2}$, IQR $=4089-4433 \mu \mathrm{m}^{2}$; Carnegie 242 stage 10 somites, median area $=4704 \mu \mathrm{m}^{2}$, IQR $=4477-5343 \mu \mathrm{m}^{2}$; see Fig 5B). 243 Together, these results suggest that our organoid-derived somite-like structures share 244 spatial and molecular features as well as overall size with their in vivo counterparts.

245 Finally, we assessed whether Somitoids can give rise to downstream paraxial 246 mesoderm derivatives of sclerotome and dermomyotome. First we differentiated 247 Somitoids to sclerotome by exposing day 5 organoids to SHH agonist and WNT inhibitor 
248 to mimic the signaling environment of ventral somites in vivo (Fan et al., 1995; Fan and 249 Tessier-Lavigne, 1994; Loh et al., 2016). After 3 days, the organoids showed a robust 250 induction of canonical sclerotome marker genes such as PAX1, SOX9 and COL2A1 251 (Fig 5C). Additionally, day 5 Somitoids were differentiated towards dermomyotome by 252 exposing them to WNT/BMP agonists and SHH inhibitor for 48 hours followed by 253 dissociation and culture on Matrigel as a monolayer in muscle differentiation medium 254 (Loh et al., 2016; Matsuda et al., 2020) to further differentiate them to skeletal muscle. 255 Immunostaining for Myosin heavy chain (MYH1, a myocyte/myotube marker) confirmed 256 that our Somitoid-derived cells can generate skeletal muscle derivatives in vitro (Figure 257 5-Supplemental Fig 1). These data demonstrate that our in vitro induced somite-like 258 structures maintain their ability to differentiate further into somitic mesoderm derivatives 259 of the sclerotome and dermomytome lineages.

\section{Discussion}

263 Here, we reported the generation of human paraxial mesoderm organoids from hPSCs 264 that reproduce important features of somitogenesis not previously captured in 265 conventional monolayer differentiation cultures, most notably formation of somite-like 266 structures. Using a simple suspension culture which does not require manual matrix 267 embedding, we identified optimal differentiation conditions by systematically screening 268 initial cell numbers and modulating the signaling factors. Importantly, our culture 269 conditions are compatible with high-throughput screening approaches. Many 270 established organoid protocols currently have limited applications because they are not 271 reproducible. Therefore, we set out to identify the optimal differentiation conditions that 272 minimized the variability between organoids as quantified by automated image analysis.

273 One critical parameter we identified in our screens was the initial cell number 274 used for aggregation. Our results suggest that if the initial cell number is above a certain 275 threshold then somite fate cannot be induced in a homogeneous manner in our 276 organoid system. This result is in line with previous findings in 3D models such as 277 gastruloids, multi-axial self-organizing aggregates of mouse ES cells, which exhibit a 
278 higher degree of variability and multiple elongations when the number of initial cells 279 exceeds a threshold (Beccari et al., 2018; van den Brink et al., 2014). Another important 280 finding of our screens was that simply removing FGF, WNT pathway agonist as well as 281 BMP inhibitor yielded the most reproducible and efficient somite-like structure-forming 282 organoids. This treatment regime does not necessarily follow from applying prior in vivo 283 and in vitro knowledge of somitogenesis. Previous protocols have used FGF and WNT 284 inhibitors (Matsuda et al., 2020) or inhibition of all four candidate signaling pathways 285 (FGF, WNT, BMP and TGF- $\beta$ ) to induce somite fate (Loh et al., 2016) in monolayer 286 cultured hiPSCs. While these conditions similarly induced somite fate marker genes in 287 our 3D system, removal of FGF/WNT agonists and BMP inhibitor overall performed 288 better as indicated by larger organoid diameters, higher average PAX3 expression 289 levels, and higher number of somite-like structures. In line with these findings, our 290 single-cell RNA-seq analysis revealed that day 5 somite-like cells from our optimized 291 protocol autonomously downregulate WNT target genes (DKK1, AXIN2, WNT3A, 292 RSPO3; Fig 4-Supplemental Fig 2) and FGF target genes (FGF8, FGF17, SPRY4, 293 DUSP6/MKP3, SEF/IL17RD; Fig 4-Supplemental Fig 5). Finally, our screening results 294 also suggest that focusing on marker gene induction as a phenotypic readout alone is 295 not sufficient to optimize culture conditions of more complex organoid models such as somitogenesis.

297 The single-cell RNA-seq analysis of our hPSC-derived Somitoids independently 298 confirmed our immunostaining and QRT-PCR results and showed that all major paraxial 299 mesodermal cell types are generated, consistent with the cell types observed during PM 300 development. Comparing our single-cell dataset with previously published in vitro 301 generated human PM transcriptomic data of monolayer cultures (Diaz-Cuadros et al., 302 2020; Matsuda et al., 2020) reveals a similar pattern of activation of marker genes. 303 Diaz-Cuadros and colleagues did not generate bona fide somitic cells as their final time 304 point cell population does not express canonical somitic markers. Matsuda et al. indeed 305 show expression of several somitic marker genes including TCF15, MEOX1, and PAX3 306 based on bulk RNA-seq data. Interestingly, our own analysis of day 5 somitic cells 307 revealed multiple distinct sub-clusters, suggesting transcriptional heterogeneity within 308 this population, which could have not been inferred from bulk data (Figure 4B,C and 
309 Figure 4-Supplemental Fig 4). Importantly, neither of these papers report formation of 310 somite-like structures, suggesting that transcriptional similarity alone is not sufficient to 311 predict morphological features, in line with our screening results showing that average 312 marker gene expression is not a good predictor of in vitro induction efficiency of somite313 like structures (Fig 3D,E and Figure 3-Supplemental Fig 2A-C).

314 While expression patterns of canonical somitic marker genes seem to be 315 conserved in humans, it will be interesting to perform detailed gene expression analysis 316 to identify putative human-specific genes of somite differentiation. Since our Somitoid 317 system is reproducible, it could serve as a versatile platform to perform functional 318 screens of human-specific or disease-relevant genes using CRISPR/Cas9 or small 319 molecule inhibitor libraries. Somitoids thus provide a powerful in vitro system for 320 studying the regulation and dynamics of human somitogenesis, including somite 321 formation.

322 One limitation of this work is that the current protocol does not produce an 323 anterior-posterior axis and therefore does not generate somite-like structures in a 324 bilaterally symmetric fashion as in the vertebrate embryo. A similar phenotype was 325 recently reported in mouse gastruloids that were embedded in matrigel to promote self326 organization into trunk-like structures (Veenvliet et al., 2020). Chemical modulation of 327 BMP and WNT signaling pathways in matrigel-embedded gastruloids resulted in 328 formation of somite-like structures arranged like a bunch of grapes, similar to what we 329 observed in our system. In standard culture conditions, gastruloids recapitulate the axial 330 organization of the embryo, which is missing in our Somitoids (Beccari et al., 2018; 331 Moris et al., 2020). To expand the patterning and morphogenetic potential of our 332 Somitoid system, our approach could be combined with a microfluidics setup to 333 generate spatio-temporally controlled morphogen gradients (Manfrin et al., 2019). In 334 summary, Somitoids provide a scalable, reproducible and easy to manipulate platform 335 to study molecular networks underlying the differentiation of paraxial mesoderm, as well 336 as the morphogenetic processes of somite formation. Furthermore, Somitoids represent 337 a promising in vitro system to study congenital diseases that are linked to the human 338 segmentation clock and somite formation, such as congenital scoliosis. 
340

341 


\section{Acknowledgements}

343 We would like to acknowledge support of the Nikon Imaging Center at Harvard Medical

344 School for image acquisition and consulting.

345

346 Declaration of Interests

347 The authors declare no competing interests.

348

349 
Materials and Methods

\begin{tabular}{|c|c|c|c|c|}
\hline \multicolumn{5}{|c|}{ Key Resources Table } \\
\hline $\begin{array}{l}\text { Reagent type } \\
\text { (species) or } \\
\text { resource }\end{array}$ & Designation & $\begin{array}{l}\text { Source or } \\
\text { reference }\end{array}$ & Identifiers & $\begin{array}{l}\text { Additional } \\
\text { information }\end{array}$ \\
\hline $\begin{array}{l}\text { Cell line (Homo } \\
\text { sapiens) }\end{array}$ & NCRM1 & $\begin{array}{l}\text { RUCDR } \\
\text { Infinite } \\
\text { Biologics }\end{array}$ & $\begin{array}{l}\text { RRID:CVCL } \\
\text { _1E71 }\end{array}$ & $\begin{array}{l}\text { hiPS cell } \\
\text { line }\end{array}$ \\
\hline $\begin{array}{l}\text { Cell line (Homo } \\
\text { sapiens) }\end{array}$ & ACTB-GFP & $\begin{array}{l}\text { Allen } \\
\text { Institute }\end{array}$ & $\begin{array}{l}\text { AICS-0016- } \\
184 \\
\text { (RRID:CVC } \\
\text { L_JM16) }\end{array}$ & $\begin{array}{l}\text { hiPS cell } \\
\text { line }\end{array}$ \\
\hline $\begin{array}{l}\text { Cell line (Homo } \\
\text { sapiens) }\end{array}$ & WTC-11 & $\begin{array}{l}\text { Coriell } \\
\text { Institute }\end{array}$ & $\begin{array}{l}\text { GM25256 } \\
\text { (RRID:CVC } \\
\text { L_Y803) }\end{array}$ & $\begin{array}{l}\text { hiPS cell } \\
\text { line }\end{array}$ \\
\hline Antibody & $\begin{array}{l}\text { anti-CDH2/N- } \\
\text { CADHERIN (rabbit } \\
\text { polyclonal) }\end{array}$ & Abcam & $a b 18203$ & $(1: 400)$ \\
\hline Antibody & $\begin{array}{l}\text { anti-PAX3 (mouse } \\
\text { monoclonal) }\end{array}$ & DSHB & Рax3-c & $(1: 250)$ \\
\hline Antibody & $\begin{array}{l}\text { anti-SOX2 (goat } \\
\text { polyclonal) }\end{array}$ & $\begin{array}{l}\text { R\&D } \\
\text { Systems }\end{array}$ & AF2018 & $(1: 200)$ \\
\hline Antibody & $\begin{array}{l}\text { anti- } \\
\text { T/BRACHYURY } \\
\text { (rabbit monoclonal) }\end{array}$ & Abcam & ab209665 & $(1: 200)$ \\
\hline Antibody & $\begin{array}{l}\text { anti-TBX6 (rabbit } \\
\text { polyclonal) }\end{array}$ & Abcam & ab38883 & $(1: 300)$ \\
\hline Antibody & $\begin{array}{l}\text { anti-TCF15 (rabbit } \\
\text { polyclonal) }\end{array}$ & Abcam & ab204045 & $(1: 50)$ \\
\hline
\end{tabular}




\begin{tabular}{|l|l|l|l|l|} 
Antibody & $\begin{array}{l}\text { anti-MYH1 (mouse } \\
\text { monoclonal) }\end{array}$ & DSHB & MF20-c & $(1: 300)$ \\
\hline $\begin{array}{l}\text { Sequence-based } \\
\text { reagent }\end{array}$ & RT-qPCR primers & & & $\begin{array}{l}\text { Supplemen } \\
\text { tal Table 2 }\end{array}$ \\
\hline $\begin{array}{l}\text { Chemical } \\
\text { compound, drug }\end{array}$ & CHIR99021 & Sigma & SML1046 & \\
\hline $\begin{array}{l}\text { Chemical } \\
\text { compound, drug }\end{array}$ & LDN193189 & Stemgent & $04-0074$ & \\
\hline $\begin{array}{l}\text { Chemical } \\
\text { compound, drug }\end{array}$ & $\begin{array}{l}\text { Y-27362 } \\
\text { dihydrochloride }\end{array}$ & Tocris & 1254 & \\
\hline
\end{tabular}

352 Human iPS cell culture and 3D differentiation

353 Human iPS cells were maintained on Matrigel-coated plates (Corning, cat no. 354277) 354 in mTeSR1 media (Stem Cell Technologies, 85870) using maintenance procedures 355 developed by the Allen Institute for Cell Science 356 (https://www.coriell.org/1/AllenCellCollection). NCRM1 iPS cells were obtained from 357 RUCDR Infinite Biologics, ACTB-GFP (cell line ID: AICS-0016 cl.184) fluorescent 358 reporter iPS cell line was obtained from the Allen Institute for Cell Science, and the 359 WTC-11 (GM25256) cell line was obtained from the Coriell Institute for Medical 360 Research. All cell lines were tested for mycoplasma contamination. We verified cell line 361 identity by immunostaining for pluripotency markers POU5F1 and SOX2.

362 For generation of paraxial mesoderm organoids, 500 dissociated iPS cells 363 resuspended in mTeSR1 media containing $10 \mu \mathrm{M}$ Y-27362 dihydrochloride (ROCKi; 364 Tocris Bioscience, cat. no. 1254) and $0.05 \%$ poly-vinyl alcohol (PVA) were dispensed 365 into 96-well U-bottom non-adherent suspension culture plates (Greiner Bio-One, 366 650185) and allowed to aggregate for 24 hours. To induce paraxial mesoderm 
367 differentiation, 24 hour old pluripotent spheroids were subjected to CL media consisting 368 of RHB Basal media (Takara/Clontech, cat. no. Y40000), 5\% KSR (Thermo Fisher 369 Scientific, cat. no. 10828028) with $10 \mu \mathrm{M}$ CHIR99021 (Sigma-Aldrich, cat. no. 370 SML1046), $0.5 \mu M$ LDN193189 (Stemgent cat. no. 04-0074), and $5 \mu M$ ROCKi for the 371 first 24 hours. Organoids were cultured in CL media without ROCKi from 24-72 hours of 372 differentiation. On day 3 (72-120 hours), CL media was supplemented with $20 \mathrm{ng} / \mathrm{ml}$ 373 FGF2 (PeproTech, cat. no. 450-33). On day 4, organoids were cultured in basal media 374 only, without the addition of signaling factors.

\section{Human sclerotome and dermomyotome differentiation}

376 To further differentiate Somitoids towards sclerotome fate, day 5 somite-stage 377 organoids were treated with $5 \mathrm{nM}$ of Shh agonist SAG $21 \mathrm{k}$ (Tocris, cat. no. 5282) and 1 $\mu \mathrm{M}$ of Wnt inhibitor C59 for 3 days as previously described (Loh et al., 2016). Organoids were subsequently differentiated towards cartilage by culturing them in the presence of $20 \mathrm{ng} / \mathrm{ml}$ BMP4 (R\&D Systems, cat. no. 314-BP-010) for 6 days.

To differentiate Somitoids towards dermomyotome, day 5 somite-stage organoids were treated with CHIR99021 ( $3 \mu \mathrm{M})$, GDC0449 (150 nM) and BMP4 (50 $\mathrm{ng} / \mathrm{ml}$ ) for 48 hours as described previously (Loh et al., 2016; Matsuda et al., 2020). In vitro skeletal muscle differentiation

Day 7 Organoids differentiated towards dermomyotome fate were dissociated with Accutase, resuspended in muscle induction medium containing ROCK inhibitor Y27632 and seeded (1.5-2.5 $\times 10^{5}$ cells per well) onto Matrigel-coated 12-well plates. To induce human skeletal muscle cells, we used a N2/horse-serum containing induction medium as previously described (Matsuda et al., 2020). In brief, DMEM/F12 containing Glutamax (Thermo Fisher Scientific, Cat. No. 10565018), 1\% insulin-transferrinselenium (Thermo Fisher Scientific, Cat. No. 41400045), 1\% N-2 Supplement (Thermo Fisher Scientific, Cat. No. 17502-048), 0.2 penicillin/streptomycin (Sigma-Aldrich, Cat. No. P4333-100ML), 2\% horse serum (Sigma-Aldrich, Cat. No. H1270-100ML). Medium was changed every other day. Day 45 cells were fixed in 4\% PFA and immunostained 395 for Myosin heavy chain (DSHB, MF20-c, 1:300). 
399 For the systematic small molecular inhibitor screen, PM organoids were generated and 400 differentiated until day 3 (PSM) of our protocol. On day 3, media was replaced with 401 fresh media containing combinations of small molecule inhibitors targeting the FGF, 402 WNT, BMP and TGF- $\beta$ signaling pathways at indicated concentrations. For targeting the 403 WNT pathway, we used C59 (Tocris, cat. no. 5148), XAV939 (Tocris, cat. no. 3748) and 404 CHIR99021 (Sigma-Aldrich, cat. no. SML1046). For inhibiting the FGF pathway we 405 used PD173074 (Sigma-Aldrich, cat. no. P2499). For inhibiting the BMP pathway we 406 used LDN193189 (Stemgent, cat. no. 04-0074). For inhibition of the TGF- $\beta$ pathway we 407 used A-83-01 (Tocris, cat. no. 2939). Media was changed daily. We analyzed 3 408 replicates per condition in the primary screen, and 5 replicates per condition in the 409 secondary screen.

410 Immunostaining

411 For organoid whole-mount immunostaining, organoids were collected in cold PBS and 412 fixed in 4\% paraformaldehyde for 1-2 hours depending on size/stage. Organoids were 413 washed in PBS and PBSFT (PBS, 0.2\% Triton X-100, 10\% FBS), and blocked in 414 PBSFT $+3 \%$ normal donkey serum. Primary antibody incubation was performed in the 415 blocking buffer overnight at $4^{\circ} \mathrm{C}$ on a rocking platform. After extensive washes in 416 PBSFT, secondary antibody incubation (1:500, all secondary antibodies were raised in 417 donkey) was performed overnight in PBSFT. Organoids were washed first in PBSFT 418 and, for the final washes, were transferred to PBT (PBS, $0.2 \%$ Triton X-100, 0.2\% BSA), 419 followed by $50 \%$ glycerol in PBT and $70 \%$ glycerol in PBT prior to mounting. Hoechst 420 (1:2000) was added to the last PBSFT wash. A list of primary antibodies is provided in 421 Table S1.

422 Confocal and time-lapse microscopy

423 All whole-mount immunostaining images were collected with a Nikon A1R point 424 scanning confocal with spectral detection and resonant scanner on a Nikon Ti-E 425 inverted microscope equipped with a Plan Apo VC 20x objective (NA 0.75). Alexa-488, 426 Alexa-594, Alexa-647 fluorophores coupled to secondary antibodies were excited with 427 the $488 \mathrm{~nm}, 561 \mathrm{~nm}$, and $647 \mathrm{~nm}$ laser lines from a Spectral Applied Research LMM-5 428 laser merge module with solid state lasers (selected with an AOTF) and collected with a 
405/488/561/647 quad dichroic mirror (Chroma). For time-lapse experiments, images were acquired with a Yokagawa CSU-X1 spinning disk confocal on a Nikon Ti inverted microscope equipped with a Plan Apo 20x objective (NA 0.75) and a Hamamatsu Flash4.0 V3 sCMOS camera. Samples were grown on 6-well glass-bottom multiwell 433 plates with No. 1.5 glass (Cellvis, cat. no. P06-1.5H-N) and mounted in a OkoLab $37^{\circ} \mathrm{C}$, $4345 \%$ CO2 cage microscope incubator warmed to $37^{\circ} \mathrm{C}$. Images were collected every 15 $435 \mathrm{~min}$, using an exposure time of $800 \mathrm{~ms}$. At each time-point, $30 \mathrm{z}$-series optical sections 436 were collected with a step-size of $2 \mu \mathrm{m}$. Multiple stage positions were collected using a 437 Prior Proscan II motorized stage. Z-series are displayed as maximum z-projections, 438 and gamma, brightness, and contrast were adjusted (identically for compared image 439 sets) using Fiji/lmageJ (Schindelin et al., 2012; https://imagej.net/Fiii).

440 Automated image segmentation and analysis

441 Automated image analysis, including background de-noising, segmentation and feature 442 extraction was done using ImageJ/Fiji macro language run in batch mode to process the 443 entire screen data set. First, binary masks were generated from the Hoechst (nuclear 444 stain) channel by de-noising the image (Gaussian Blur, sigma=5) followed by applying 445 Li's Minimum Cross Entropy thresholding method (Li and Tam, 1998) and refining binary 446 masks through several rounds of erosion/dilation steps. Next, binary masks were 447 converted to selections and added to the Region of Interest (ROI) Manager. Finally, 448 ROls were used to perform diameter measurements of organoids. For Pax3 449 measurements, Hoechst and Pax3 channels were first denoised using a Gaussian blur 450 filter (sigma $=10$ ) and then used to create a normalized Pax3 image by dividing the Pax3 451 channel with the Hoechst channel. Next, ROls based on Hoechst binary masks were 452 applied to the Pax3 normalized image to extract fluorescence intensity measurements 453 for each z-slice. Finally, mean Pax3 intensity values for each organoid were calculated 454 and compared.

455 Quantification of per organoid number of somite-like structures for secondary 456 screen

457 For the primary and secondary screens, images were acquired on a Nikon A1R point 458 scanning confocal microscope. For each organoid, 66 z-series optical sections were 459 collected with a step-size of $2 \mu \mathrm{m}$. Quantification of somite-like structures for the 
secondary screen was done by blinded manual scoring, considering the following 461 criteria:

462 1. Nuclear expression of somitic marker PAX3.

463 2. Accumulation of NCAD around a central cavity.

464 3. Radial arrangement of PAX3+ columnar cells around the central cavity (rosette-like 465 structure).

\section{Quantification of organoid and human somite sizes}

467 Carnegie stage 9, 10 and 11 human embryonic somite data was obtained from the 468 Virtual Human Embryo Project (https://www.ehd.org/virtual-human-embryo/). Somite 469 sizes of human embryos were measured using the Ruler Tool on the Virtual Human 470 Embryo website along the medio-lateral and dorso-ventral axis of the embryo. The slice 471 with the largest diameter of each somite was used for measurements. Sizes of somite472 like structures of day 5 organoids were measured along the $X$ and $Y$ axes of the image 473 since, unlike in the embryo, they do not exhibit morphological anisotropies. Somite 474 areas were approximated by using the two diameter measurements from each somite475 like structure to calculate the area of the resulting rectangle.

\section{RNA extraction, reverse transcription and qPCR}

477 Organoids were harvested in Trizol (Life Technologies cat. no. 15596-018), followed by 478 precipitation with Chloroform and Ethanol and transfer onto Purelink RNA Micro Kit 479 columns (Thermo Fisher cat. no. 12183016) according to manufacturer's protocol, 480 including on-column DNase treatment. A volume of $22 \mu \mathrm{l}$ RNase-free water was used 481 for elution and RNA concentration was measured with a Qubit Fluorometer. Typically, 482 between $0.2-1 \mu \mathrm{g}$ of RNA was reverse transcribed using Superscript III First Strand 483 Synthesis kit (Life Technologies cat. no. 18080-051) and oligo-dT primers to generate 484 cDNA libraries.

485 For real time quantitative PCR, cDNA was diluted 1:30-1:50 in water and qPCR 486 was performed using the iTaq Universal SYBR Green kit (Bio-Rad cat. no. 1725124). 487 Each gene-specific primer and sample mix was run in triple replicates. Each $10 \mu \mathrm{l}$ 488 reaction contained $5 \mu \mathrm{l}$ 2X SYBR Green Master Mix, $0.4 \mu \mathrm{l}$ of $10 \mu \mathrm{M}$ primer stock $(1: 1$ 489 mix of forward and reverse primers), and $4.6 \mu \mathrm{l}$ of diluted cDNA. qPCR plates were run 490 on a Roche LightCycler 480 Real-Time PCR system with the following cycling 
491 parameters: initial denaturation step $\left(95^{\circ} \mathrm{C}\right.$ for 1 minute), 40 cycles of amplification and 492 SYBR green signal detection (denaturation at $95^{\circ} \mathrm{C}$ for 5 seconds, annealing/extension 493 and plate read at $60^{\circ} \mathrm{C}$ for 40 seconds), followed by final rounds of gradient annealing 494 from $65^{\circ} \mathrm{C}$ to $95^{\circ} \mathrm{C}$ to generate dissociation curves. Primer sequences are listed in Table 495 S2. All unpublished primers were validated by checking for specificity (single peak in 496 melting curve) and linearity of amplification (serially diluted cDNA samples). For relative 497 gene expression analysis, the $\Delta \Delta \mathrm{Ct}$ method was implemented using the $\mathrm{R}$ package ' $p c r$ ' 498 (https://cran.r-project.org/web/packages/pcr/). PP1A was used as the housekeeping 499 gene in all cases. Target gene expression is expressed as fold change relative to 500 undifferentiated iPS cells.

\section{Preparation of single-cell suspensions for scRNA-seq}

502 Cell dissociation protocols were optimized to achieve single cell suspensions with $>90 \%$ 503 viable cells and low number of doublets.

504 Organoids collected at day $1,2,3$, and 5 of our differentiation protocol were 505 pooled in pre-warmed PBS, transferred to pre-warmed accutase and incubated for 5-7 $506 \min$ at $37^{\circ} \mathrm{C}$. For Day 1 organoid cell suspension, 30 organoids were pooled. For Day 2 507 organoid cell suspension, 15 organoids were pooled. For Day 3 cell suspension, 8 508 organoids were pooled. For Day 5 cell suspension, 5 organoids were pooled. Organoids 509 were briefly rinsed in PBS, then transferred to $500 \mu \mathrm{PBS} / 0.05 \% \mathrm{BSA}$ and carefully 510 triturated to generate a single-cell suspension. Cell suspension was run through a cell 511 strainer (Falcon, cat. no. 352235) and transferred to a $1.5 \mathrm{ml}$ tube. Cells were spun 512 down at $250 \mathrm{~g}$ for $3 \mathrm{~min}$ at $4^{\circ} \mathrm{C}$. Cell pellet was resuspended in $25 \mu \mathrm{lPB} / 0.05 \% \mathrm{BSA}$, 513 cell concentration and viability was measured using an automated cell counter, and cell 514 suspension was further diluted as appropriate to reach the optimal range for $10 \times$ (7005151200 cells per $\mu$ l). Cells were subjected to single-cell RNA sequencing (10x Genomics, 516 Chromium Single Cell 3' v3) aiming for the following target cell numbers:

517 Day 1, 3,000 cells; Day 2, 4,000 cells; Day 3, 5,000 cells; Day 5, 6,000 cells. Estimated 518 actual cell numbers collected were: Day 1, 2,930 cells; Day 2, 4,977; Day 3, 5,968 cells; 519 Day 5, 4,841 cells. Single-cell libraries were generated using standard protocols. 520 Libraries were sequenced together on a NovaSeq 6000 system resulting in 800 million 521 reads. 


\section{Analysis of scRNA-seq data}

529 Statistics and plots were generated using R version 4.0.2 "Taking Off Again" and Seurat 530 version 3.0 (Stuart et al., 2019).

\section{QC analysis / processing of scRNA-seq data}

532 Cell Ranger pipeline (10x Genomics, Version 4.0.0) was used to de-multiplex the raw 533 base call files, generate FASTQ files, perform the alignment against the human 534 reference genome (GRCh38 1.2.0), and generate the count matrices.

535 For the initial QC, we determined the following thresholds for filtering out low-quality 536 cells: UMI counts less than 500, gene counts less than 200, mitochondrial fraction 537 above 0.2 and a complexity score of less than 0.8 (calculated as $538 \log 10$ (genes)/log10(UMIs)).

\section{Low-dimensional embedding and clustering}

540 After QC filtering, we normalized our dataset using the sctransform (Hafemeister and 541 Satija, 2019) framework, which is part of the Seurat package. To regress out 542 confounding variation in our dataset, we performed cell cycle scoring and determined 543 mitochondrial mapping percentage using standard workflows. Next, we performed PCA 544 and determined the K-nearest neighbor graph using the first 40 principle components. 545 We then applied the Leiden clustering algorithm using a parameter range from 0.1-1.0 546 to determine the best resolution/number of clusters, which reflected biological 547 differences (FindClusters, resolution=0.1-1.0). Clusters were visualized on a UMAP 548 embedding (RunUMAP, dims $=1: 40$ ). To determine optimal resolution for clustering and 549 assign cell types for each cluster, we visualized sets of known marker genes for each 550 predicted cell type on UMAP plots. Prior to marker gene identification and final 551 assignments of cluster identities, we also checked additional quality control metrics 552 (UMI count, gene count, mitochondrial gene ratio) to exclude low-quality clusters from 
553 downstream analyses. Through iterative analysis we determined Leiden clustering with 554 resolution $=0.8$, resulting in 22 clusters, to best capture biological variation of the 555 dataset. Using a combination of quality control metrics and unbiased marker gene 556 identification for each cluster (see below), we excluded seven smaller low-quality 557 clusters (as determined by QC metrics and/or expression of stress signature genes)

558 from further downstream analysis (15 clusters after filtering).

\section{Identification of differentially expressed genes}

561 Marker genes for every cluster were identified by a two-sided Wilcoxon rank-sum test 562 comparing cells from each cluster to all other cells in the combined dataset. Genes were 563 considered differentially expressed if the log2 fold-change average expression in the 564 cluster is equal or greater 0.25 relative to the average expression in all other clusters 565 combined, and the adjusted P-value <0.05. Multiple comparison correction was 566 performed using the Bonferroni method. Identified marker genes for the top 20 567 differentially expressed transcripts are listed in Fig 4-Supplemental Fig 4A. The full list 568 of differentially expressed genes, ranked by adjusted P-values and associated fold569 changes are provided in Supplemental Table 1. 
Aulehla A, Wehrle C, Brand-Saberi B, Kemler R, Gossler A, Kanzler B, Herrmann BG. 2003. Wnt3a plays a major role in the segmentation clock controlling somitogenesis. Dev Cell 4:395-406. doi:10.1016/S1534-5807(03)00055-8 Aulehla A, Wiegraebe W, Baubet V, Wahl MB, Deng C, Taketo M, Lewandoski M, Pourquié $O$. 2008. A beta-catenin gradient links the clock and wavefront systems in mouse embryo segmentation. Nat Cell Biol 10:186-193. doi:10.1038/ncb1679 Beccari L, Moris N, Girgin M, Turner DA, Baillie-Johnson P, Cossy A-C, Lutolf MP, Duboule D, Arias AM. 2018. Multi-axial self-organization properties of mouse embryonic stem cells into gastruloids. Nature 562:272-276. Chal J, Oginuma M, Al Tanoury Z, Gobert B, Sumara O, Hick A, Bousson F, Zidouni $Y$, Mursch $C$, Moncuquet $P$, Tassy $O$, Vincent $S$, Miyanari A, Bera A, Garnier J-M, Guevara G, Hestin M, Kennedy L, Hayashi S, Drayton B, Cherrier T, Gayraud-Morel B, Gussoni E, Relaix F, Tajbakhsh S, Pourquié O. 2015. Differentiation of pluripotent stem cells to muscle fiber to model Duchenne muscular dystrophy. Nat Biotechnol 33:962-969. doi:10.1038/nbt.3297

Chal J, Pourquié O. 2017. Making muscle: skeletal myogenesis in vivo and in vitro. Development 144:2104-2122.

Chu L-F, Mamott D, Ni Z, Bacher R, Liu C, Swanson S, Kendziorski C, Stewart R, Thomson JA. 2019. An In Vitro Human Segmentation Clock Model Derived from Embryonic Stem Cells. Cell Rep 28:2247-2255.e5. doi:10.1016/j.celrep.2019.07.090

Delfini M-C, Dubrulle J, Malapert P, Chal J, Pourquié O. 2005. Control of the segmentation process by graded MAPK/ERK activation in the chick embryo. Proceedings of the National Academy of Sciences 102:11343-11348. doi:10.1073/pnas.0502933102 Dequeant ML, Glynn E, Gaudenz K, Wahl M, Chen J, Mushegian A, Pourquie O. 2006. A Complex Oscillating Network of Signaling Genes Underlies the Mouse Segmentation Clock. Science 314:1595-1598. doi:10.1126/science.1133141 Diaz-Cuadros M, Wagner DE, Budjan C, Hubaud A, Tarazona OA, Donelly S, Michaut A, Al Tanoury Z, Yoshioka-Kobayashi K, Niino Y, Kageyama R, Miyawaki A, Touboul J, Pourquié O. 2020. In vitro characterization of the human segmentation clock. Nature 580:113-118. doi:10.1038/s41586-019-1885-9 Dubrulle J, McGrew MJ, Pourquié O. 2001. FGF Signaling Controls Somite Boundary Position and Regulates Segmentation Clock Control of Spatiotemporal Hox Gene Activation. Cell 106:219-232. doi:10.1016/S0092-8674(01)00437-8 Dunty WC Jr, Biris KK, Chalamalasetty RB, Taketo MM, Lewandoski M, Yamaguchi TP. 2008. Wnt3a/beta-catenin signaling controls posterior body development by coordinating mesoderm formation and segmentation. Development 135:85-94. 

Long-range sclerotome induction by sonic hedgehog: direct role of the aminoterminal cleavage product and modulation by the cyclic AMP signaling pathway. Cell 81:457-465. doi:10.1016/0092-8674(95)90398-4 Fan CM, Tessier-Lavigne M. 1994. Patterning of mammalian somites by surface ectoderm and notochord: evidence for sclerotome induction by a hedgehog homolog. Cell 79:1175-1186. doi:10.1016/0092-8674(94)90009-4 Freedman BS, Brooks CR, Lam AQ, Fu H, Morizane R, Agrawal V, Saad AF, Li MK, Hughes MR, Werff RV, Peters DT, Lu J, Baccei A, Siedlecki AM, Valerius MT, Musunuru K, McNagny KM, Steinman TI, Zhou J, Lerou PH, Bonventre JV. 2015. Modelling kidney disease with CRISPR-mutant kidney organoids derived from human pluripotent epiblast spheroids. Nat Commun 6:8715. doi: $10.1038 /$ ncomms 9715 Greco TL, Takada S, Newhouse MM, McMahon JA, McMahon AP, Camper SA. 1996. Analysis of the vestigial tail mutation demonstrates that Wnt-3a gene dosage regulates mouse axial development. Genes Dev 10:313-324. doi:10.1101/gad.10.3.313 Hafemeister C, Satija R. 2019. Normalization and variance stabilization of singlecell RNA-seq data using regularized negative binomial regression. bioRxiv. doi:10.1101/576827 Hubaud A, Pourquié O. 2014. Signalling dynamics in vertebrate segmentation. Nat Rev Mol Cell Biol 15:709-721. $\mathrm{Li} \mathrm{CH}$, Tam PKS. 1998. An iterative algorithm for minimum cross entropy thresholding. Pattern Recognit Lett 19:771-776. doi:10.1016/S01678655(98)00057-9 REA, Chen Z, Vogel H, Epstein JA, Kundaje A, Talbot WS, Beachy PA, Ang LT, Weissman IL. 2016. Mapping the Pairwise Choices Leading from Pluripotency to Human Bone, Heart, and Other Mesoderm Cell Types. Cell 166:451-467. doi:10.1016/j.cell.2016.06.011 Manfrin A, Tabata Y, Paquet ER, Vuaridel AR, Rivest FR, Naef F, Lutolf MP. 2019. Engineered signaling centers for the spatially controlled patterning of human pluripotent stem cells. Nat Methods 16:640-648. doi:10.1038/s41592-019-0455-2 Matsuda M, Yamanaka Y, Uemura M, Osawa M, Saito MK, Nagahashi A, Nishio M, Guo L, Ikegawa S, Sakurai S, Kihara S, Maurissen TL, Nakamura M, Matsumoto T, Yoshitomi H, Ikeya M, Kawakami N, Yamamoto T, Woltjen K, Ebisuya M, Toguchida J, Alev C. 2020. Recapitulating the human segmentation clock with pluripotent stem cells. Nature 580:124-129. doi:10.1038/s41586-020-2144-9 
Moris N, Anlas K, van den Brink SC, Alemany A, Schröder J, Ghimire S, Balayo T, van Oudenaarden A, Martinez Arias A. 2020. An in vitro model of early anteroposterior organization during human development. Nature 582:410-415. doi:10.1038/s41586-020-2383-9 Oates AC, Morelli LG, Ares S. 2012. Patterning embryos with oscillations: structure, function and dynamics of the vertebrate segmentation clock. Development 139:625-639. doi:10.1242/dev.063735 Palla A, Blau H. 2020. The clock that controls spine development modelled in a dish. Nature 580:32-34. doi:10.1038/d41586-020-00322-y Sakurai H, Sakaguchi Y, Shoji E, Nishino T, Maki I, Sakai H, Hanaoka K, Kakizuka A, Sehara-Fujisawa A. 2012. In vitro modeling of paraxial mesodermal progenitors derived from induced pluripotent stem cells. PLoS One 7:e47078. doi:10.1371/journal.pone.0047078 Schindelin J, Arganda-Carreras I, Frise E, Kaynig V, Longair M, Pietzsch T, Preibisch S, Rueden C, Saalfeld S, Schmid B, Tinevez J-Y, White DJ, Hartenstein V, Eliceiri K, Tomancak P, Cardona A. 2012. Fiji: an open-source platform for biological-image analysis. Nat Methods 9:676-682. doi:10.1038/nmeth.2019 Stuart T, Butler A, Hoffman P, Hafemeister C, Papalexi E, Mauck WM, Hao Y, Stoeckius M, Smibert P, Satija R. 2019. Comprehensive Integration of Single-Cell Data. Cell 0. Tan JY, Sriram G, Rufaihah AJ, Neoh KG, Cao T. 2013. Efficient derivation of lateral plate and paraxial mesoderm subtypes from human embryonic stem cells through GSKi-mediated differentiation. Stem Cells Dev 22:1893-1906. Tonegawa A, Funayama N, Ueno N, Takahashi Y. 1997. Mesodermal subdivision along the mediolateral axis in chicken controlled by different concentrations of BMP-4. Development 124:1975-1984. Traag VA, Waltman L, van Eck NJ. 2019. From Louvain to Leiden: guaranteeing well-connected communities. Sci Rep 9:5233. doi:10.1038/s41598-019-41695-z Tzouanacou E, Wegener A, Wymeersch FJ, Wilson V, Nicolas J-F. 2009. Redefining the progression of lineage segregations during mammalian embryogenesis by clonal analysis. Dev Cell 17:365-376. van den Brink SC, Baillie-Johnson P, Balayo T, Hadjantonakis A-K, Nowotschin S, Turner DA, Martinez Arias A. 2014. Symmetry breaking, germ layer specification and axial organisation in aggregates of mouse embryonic stem cells. Development 141:4231-4242. doi:10.1242/dev.113001 F, Pustet M, Heimann S, Buschow R, Wittler L, Timmermann B, Meissner A, Herrmann BG. 2020. Mouse embryonic stem cells self-organize into trunk-like structures with neural tube and somites. bioRxiv. doi:10.1101/2020.03.04.974949 Xi H, Fujiwara W, Gonzalez K, Jan M, Liebscher S, Van Handel B, Schenke- 
$690 \quad$ Layland K, Pyle AD. 2017. In Vivo Human Somitogenesis Guides Somite 691 Development from hPSCs. CellReports 18:1573-1585.

692 Yamaguchi TP, Takada S, Yoshikawa Y, Wu N, McMahon AP. 1999. T (Brachyury) 693 is a direct target of Wnt3a during paraxial mesoderm specification. Genes \& $694 \quad$ Development. doi:10.1101/gad.13.24.3185

695 
697 Figure 1. Human PSC-derived paraxial mesoderm organoids turn on marker 698 genes associated with paraxial mesoderm differentiation.

(A) Schematic overview of paraxial mesoderm (PM) organoid differentiation protocol from human pluripotent stem cells (PSCs). Human PSCs aggregated and formed spheroids for 24 hours prior to differentiation. For differentiation, spheroids were exposed to Wnt agonist (CHIR) and BMP inhibitor (LDN) for 72 hours. On day 3, FGF2 was added to the media in addition to CHIR and LDN.

(B) Immunofluorescence analysis of cell fate-specific marker genes shows progressive differentiation towards PSM fate (top and middle row). Organoids derived from human iPS cells harboring an MSGN1-Venus reporter express TBX6 at the same time as the reporter is activated (bottom row). Scale bar represents $100 \mu \mathrm{m}$. Representative images shown from $\mathrm{n}=3$ independent experiments. Cell lines used: NCRM1 hiPSCs and MSGN1-Venus hiPS reporter cells.

(C) qRT-PCR analysis of PSM and Somite markers reveals PSM-to-somite transition from day 4 to day 5 . Relative gene expression levels are shown as Z-scores, expressed as fold-change relative to undifferentiated iPS cells (see Methods). Source data is available in Figure 1-Source Data 1. 
Figure 2. Pilot screen to optimize differentiation conditions for somite phenotype 718 in PM organoids reveals optimal initial number of cells and duration of treatment.

(A) Schematic overview of systematic screen in PM organoids (Somitoids). PSMagonists/antagonists. Treated organoids were cultured in basal media with inhibitors as indicated. Control organoids were maintained in CL media with FGF added. NCRM1-derived organoids were used for the screen.

(B) Representative immunofluorescent images of day 4 and day 5 organoids after treatment for 24 hours or 48 hours, respectively, stained for somite marker PAX3 and F-ACTIN to visualize rosette-like somite structures. Organoids generated from 1000 cells generally show a more diffuse F-ACTIN pattern compared to organoids made from 500 cells, which exhibit bright foci, consistent with somite formation. Confocal images are shown as maximum intensity z-projections. Scale bar represents $100 \mu \mathrm{m}$. Small molecule inhibitors used are indicated in brackets. FGFRi: FGF receptor inhibitor (PD173074). WNTi: Wnt inhibitor (C59 or XAV939). Bi: BMP inhibitor (LDN). TGF- $\beta i$ : TGF- $\beta$ inhibitor (A-83-01). Representative image shown for each condition from 3 organoid replicates.

(C) Automated quantification of organoid diameter for each organoid/replicate treated as indicated (see Methods for details). Three organoids per condition were characterized except where indicated with grey boxes. Organoids initiated from 500 cells show a decreased diameter when treated for 48 hours compared with 24 hours. Source data is available in Fig 2-Source Data 1.

(D) Automated quantification of normalized average PAX3 intensity for each organoid/replicate treated as indicated. Three organoids are shown per condition except where indicated with grey boxes. Organoids initiated from both 500 and 1000 cells show higher average normalized PAX3 levels when treated for 24 hours compared with 48 hours. Source data is available in Fig 2-Source Data 2. 
Figure 3. Secondary screen of PM organoids identifies optimal differentiation protocol for somite formation.

(A) Schematic overview of secondary screen in PM organoids. PSM-stage organoids were treated on day 3 for 24 hours followed by measurement, 24 hours of treatment followed by 24 hour culture in basal media (no added factors) and then measured $(24 h+24 h)$, and 48 hours of treatment followed by measurement. Treated organoids were cultured in basal media with inhibitors as indicated. WNT and FGF inhibitors were tested at two different concentrations. Control organoids were maintained in CL media with FGF added. NCRM1-derived organoids were used for the screen.

(B) Representative immunofluorescent images of day 5 organoids stained for somite markers PAX3 and NCAD showing the rosette-like structures which were scored as somite-like structures based on expression of somite fate markers and structural features (scoring criteria detailed in Methods section). Images are shown as individual z-sections.

(C) Representative immunofluorescent images of day 4 and day 5 organoids stained for somite markers PAX3 and NCAD to visualize rosette-like somite structures. Confocal images are shown as maximum intensity z-projections. Scale bar represents $100 \mu \mathrm{m}$. Small molecule inhibitors used are indicated in brackets. FGFRi: FGF receptor inhibitor (PD173074). WNTi: Wnt inhibitor (C59 or XAV939). Bi: BMP inhibitor (LDN). TGF- $\beta i$ : TGF- $\beta$ inhibitor (A-83-01). Representative image shown for each condition from 5 organoid replicates.

(D) Automated quantification of normalized average PAX3 intensity for each organoid/replicate treated as indicated (see Methods for details). Five organoids are shown per condition except as indicated with grey boxes. Several inhibitor combinations with a treatment regime of 24 hour treatment followed by 24 hour cultured in basal media show highest average PAX3 levels. Source data is available in Fig 3-Source Data 1.

(E) Quantification of the number of somite-like structures for each condition. Each row represents one organoid replicate. Five organoids are shown per condition except where indicated with grey boxes. Organoids which were maintained in $\mathrm{CL}$ 
media with added FGF for 24 hours followed by culture in basal media for 24 hours reproducibly exhibit the highest number of somite-like structures per organoid. Source data is available in Fig 3-Source Data 2.

Figure 4. Single-cell RNA sequencing analysis of PM organoids (Somitoids) reveals differentiation trajectory from NMp-like cells to somite-stage paraxial mesoderm.

(A) UMAP (Uniform Manifold Approximation and Projection) of single-cell transcriptomes of differentiating human PM organoids, colored by collection time point (15,558 cells). NCRM1-derived organoids were used to collect single cells.

(B) UMAP of human PM organoids, colored by assigned Leiden cluster identity based on marker gene expression profile (see Methods).

(C) Heat map of selected marker genes of paraxial mesoderm differentiation. Collection time point and Leiden cluster identities are indicated. Marker genes are grouped based on primary associated cell fate as indicated.

(D) Heat map of single-cell HOX gene expression levels. Cells are grouped by Leiden cluster identity. Hox genes are ordered by position, with anatomical positions of HOX paralogues indicated on the right.

(E) UMAP plots overlaid with normalized transcript counts of representative cell fate marker genes. 

differentiate to sclerotome fate.

(A) Whole-mount Immunofluorescence analysis of day 5 Somitoids reveals coexpression of somite markers TCF15/PARAXIS and PAX3 and polarized rosettelike structures as indicated by F-ACTIN localization, suggesting that somite-like structures resemble in vivo counterparts in both a molecular and morphological manner. Representative images are shown as maximum intensity z-projections from 3 organoid replicates. NCRM1 hiPS cells were used to generate Somitoids.

(B) Quantification of somite-like structure sizes in day 5 human Somitoids and human embryos (Carnegie stage 9,10 and 11) reveals that the median and interquartile range of in vitro somite-like structure sizes (calculated as area) is comparable to Carnegie stage 11 human somites in vivo (see Methods section). Boxes indicate interquartile range (25th percentile to 75 th percentile). End of whiskers indicate minimum and maximum. Points indicate individual somite-like structures. Central lines represent the median. Carnegie embryo data were obtained from the Virtual Human Embryo Project (https://www.ehd.org/virtualhuman-embryo). NCRM1 hiPS cells were used to generate Somitoids. Source data is available in Fig 5-Source Data 1.

825

(C) Sclerotome differentiation of Somitoids. Day 5 Somitoids were exposed to SHH agonist and WNT inhibitors to induce sclerotome differentiation as indicated. qPCR analysis of somite and sclerotome markers reveals induction of sclerotome markers on Day 8. Relative gene expression levels are shown as Z-scores, expressed as fold-change relative to undifferentiated iPS cells (see Methods). NCRM1 hiPS cells were used to generate Somitoids. Source data is available in Fig 5-Source Data 2. 
Figure 1-Supplemental Figure 1. Additional immunofluorescent and qPCR data

Figure 1-Supplemental Figure 2. Organoids generated using an unoptimized protocol exhibit heterogeneous activation of somite marker genes (PAX3 and NCAD) and a low number of rosette structures.

843 addition, organoids generated using the unoptimized protocol showed a delayed onset

844 of PAX3 expression and the rosette structures did not form until day 8 . 
Figure 2-Supplemental Figure 1. Replicate data of pilot screen for somite phenotype in human PM organoids

(A) Schematic overview of systematic screen in PM organoids. PM-stage organoids

Figure 2-Supplemental Figure 2. Additional immunofluorescent data and interorganoid phenotypic variance of primary somite phenotype screen in Somitoids

874

875

(A) Comparison of organoids made from 500 and 1000 cells, respectively, immunostained for PAX3 (somite fate) and SOX2 (neural fate). Organoids made from 1000 cells contain patches of PAX3-negative/SOX2-positive cells (marked by dashed contours), indicating a higher degree of heterogeneity of somite fate induction. Representative images are shown from 3 organoid replicates.

(B) Heatmaps of diameter and PAX3 inter-organoid variation for each treatment, expressed as coefficient of variation (CV, in percentage). $\mathrm{CV}$ is calculated across the 2 or 3 organoid replicates of each condition as (standard deviation/mean) ${ }^{*} 100$. 
877 Figure 2-Supplemental Figure 3. Organoids made from 500 cells more 878 reproducibly generate somite-like structures compared to organoids made from 8791000 cells.

880 Representative single z-sections of organoids treated for 24 hours with inhibitors as 881 indicated. Organoids were stained for PAX3 and F-ACTIN to visualize somite-like 882 structures. Scale bar represents $100 \mu \mathrm{m}$.

883 (A) Organoids made from 500 and 1000 cells, respectively, were treated with FGFR 884 inhibitor PD173 for 24 hours on day 3 of the differentiation protocol. Two representative organoids are shown for each cell number. Organoids made from 500 cells consistently show more somite-like structures compared to organoids made from 1000 cells.

(B) Organoids made from 500 and 1000 cells, respectively, were treated with WNT inhibitor C59 for 24 hours on day 3 of the differentiation protocol. Two representative organoids are shown for each cell number. Organoids made from 500 cells show more uniform expression of PAX3 and a higher number of somitelike structures compared to organoids made from 1000 cells. 
Figure 3-Supplemental Figure 1. High-resolution imaging of somite-like structures in day 5 organoids

(A) Single z-section of a day 5 organoid treated with $C L+F G F 2$ on day 3 to day 4 ,

Figure 3-Supplemental Figure 2. Additional qPCR data and inter-organoid phenotypic variance of secondary somite phenotype screen in somitoids

(B) Z-projection of a day 5 organoid treated with $\mathrm{CL}+\mathrm{FGF2}$ on day 3 to day 4 , NCAD. ( $A^{\prime}$ ) shows an enlargement of the dashed square indicated in $(A)$ with two somite-like structures visible based on NCAD expression. (A") Merged image of the PAX3 and Hoechst (nuclear stain) channels with a dashed outline marking one of the somite-like structures.

\begin{abstract}
followed by culture in basal media for 24 hours (optimized protocol). 5
\end{abstract} consecutive z-sections ( $1 \mu \mathrm{m}$ step size) were used to generate the projection. Somite-like structures were visualized by immunostaining using somite markers PAX3 and NCAD. Scale bars indicate $50 \mu \mathrm{m}$.

(A) Quantification of the number of somite-like structures for each condition. Number of somite-like structures are shown as calculated means based on quantifying somite-like structures for 2-5 organoids per condition.

(B) Heatmaps of Somite-like structure counts and PAX3 inter-organoid variation for each treatment, expressed as coefficient of variation ( $C V$, in percentage). $C V$ is calculated across the 3-5 replicates of each condition as (standard deviation/mean)*100.

(C) qRT-PCR analysis of somite marker genes for select treatment conditions from the secondary somite phenotype screen. Expression of somite marker genes correlates well with Pax3 immunostainings from the secondary screen (see Fig 3B). Relative gene expression levels are shown as Z-scores, expressed as foldchange relative to undifferentiated iPS cells (see Methods). Source data is available in Fig 3-Supplemental Fig 2-Source Data 1. 
Figure 3-Supplemental Figure 3. The optimized Somitoid protocol is reproducible across experiments and different cell lines.

928 Quantification of the number of somite-like structures for each condition. Each row 929 represents one organoid replicate. 10 organoids are shown per condition. Optimized 930 protocol corresponds to treatment with $\mathrm{CL}+\mathrm{FGF} 2$ for 24 hours followed by culture in basal media for 24 hours. Control organoids were treated with CL+FGF2 for 48 hours.

(A) Organoids treated with the optimized protocol exhibit a robust and reproducible somite phenotype across two independent experiments (Experiment 1, average number of somite-like structures $=39+/-8($ mean $+/$-std $)$; Experiment 2 , average number of somite-like structures $=42+/-4 ; p$-val $=0.16$ ). Source data is available in Fig 3-Supplemental Fig 3-Source Data 1.

(B) Applying the optimized protocol to multiple cell lines confirms that the observed somite phenotype is reproducible across different cell lines. Two cell lines (ACTB-GFP and WTC) were characterized in addition to our screening cell line (NCRM1). NCRM1, average number of somite-like structures $=43+/-4$ (mean $+/-$ std); ACTB-GFP, average number of somite-like structures $=40+/-6$; WTC, average number of somite-like structures $=33+/-4$. One additional experimental condition ( $\left.559^{\text {hi }}, 48 \mathrm{~h}\right)$, which scored amongst the best conditions in our secondary screen, was also tested and compared across cell lines. Although the C59 treatment (a WNT inhibitor) resulted in a higher number of somite-like structures in the ACTB-GFP cell line (average number of somite-like structures = 32+/-4 (mean+/-std)) compared with the other cell lines (NCRM1, average number of somite-like structures $=20+/-3$; WTC, average number of somite-like structures $=17+/-4)$, the optimized protocol resulted in the highest number of somite-like structures in all cell lines. Source data is available in Fig 3Supplemental Fig 3-Source Data 2. 
954 Figure 4-Supplemental Figure 1. Single-cell RNA sequencing analysis of 955 differentiating human PM organoids, NMp marker genes

956 UMAP plots of single-cell transcriptomes of differentiating human PM organoids overlaid 957 with normalized transcript counts of selected marker genes. NMp, neuro-mesodermal 958 progenitor, EMT, epithelial-to-mesenchymal transition.

Figure 4-Supplemental Figure 2. Single-cell RNA sequencing analysis of differentiating human PM organoids, PSM marker genes UMAP plots of single-cell transcriptomes of differentiating human PM organoids overlaid with normalized transcript counts of selected marker genes. PSM, pre-somitic 964 mesoderm.

Figure 4-Supplemental Figure 3. Single-cell RNA sequencing analysis of differentiating human PM organoids, Somite marker genes UMAP plots of single-cell transcriptomes of differentiating human PM organoids overlaid with normalized transcript counts of selected marker genes.

Figure 4-Supplemental Figure 4. Single-cell RNA sequencing analysis of differentiating human PM organoids, cluster-based marker gene identification and HOX gene analysis

(A) Top 20 positively enriched genes for identified Leiden clusters relative to all other clusters. Genes were identified by a two-sided Wilcoxon rank-sum test and are ranked by adjusted $\mathrm{P}$ values based on Bonferroni correction. See Supplemental Table 1 for a complete list of identified marker genes for each cluster, adjusted Pvalues and fold-change values.

(B) Heat map of single-cell HOX gene expression levels. Cells are grouped by collection time point. Hox genes are ordered by position, with anatomical positions of HOX paralogues indicated on the right. 
Figure 4-Supplemental Figure 5. Single-cell RNA sequencing analysis of 985 differentiating Somitoids reveals downregulation of WNT, FGF, and NOTCH target genes in day 5 somite-like cells

987 Heat map of single-cell signaling target gene expression levels. Cells are grouped by 988 collection time point. Genes are grouped based on their signaling pathway, indicated on 989 the left. Day 5 somitic cells autonomously downregulate many WNT, FGF, and NOTCH 990 signaling target genes.

Figure 5-Supplemental Figure 1. Differentiation of Somitoid-derived cells towards 993 skeletal muscle

(A) Day 5 somite-stage organoids were further differentiated towards skeletal muscle. Organoids were first exposed to signaling modulators of WNT, $\mathrm{SHH}$, and BMP4 for 48 hours to differentiate them to dermomyotome. Next, organoids were dissociated, and further differentiated as monolayer cultures on Matrigel in muscle differentiation medium. Day 5 control organoids were cultured for an additional 48 hours in basal media (day 5-7) and not pulse-treated with WNT+/SHH-/BMP4+ prior to dissociation. Day 45 cells were stained for Myosin heavy chain (MYH1), which is expressed in myocytes, myotubes and skeletal myofibers.

(B) Day 45 cells derived from Somitoids were fixed and stained for Myosin heavy chain (MYH1) and Hoechst (nuclear stain). Somite-stage organoids (Day 5) were treated with WNT+/SHH-/BMP4+ signaling modulators for $48 \mathrm{~h}$ from day 5 to day 7.

(C) Same as in (B) but with control cells that were not pulse-treated with WNT+/SHH/BMP4+ signaling modulators but instead cultured in basal media from day 5 to day 7. 
1011 List of accompanying files

1012 Supplemental Figures

1013 Figure 1-Figure Supplement 1: Additional immunofluorescent and qPCR data

1014 Figure 1-Figure Supplement 2: Organoids generated using an unoptimized protocol 1015 exhibit heterogeneous activation of somite marker genes and a low number of rosette 1016 structures

1017 Figure 2-Figure Supplement 1: Replicate data of pilot screen for somite phenotype in 1018 human PM organoids

1019 Figure 2-Figure Supplement 2: Additional immunofluorescent data and inter-organoid 1020 phenotypic variance of primary somite phenotype screen in human PM organoids

1021 Figure 2-Supplemental Figure 3: Organoids made from 500 cells more reproducibly 1022 generate somite-like structures compared to organoids made from 1000 cells

1023 Figure 3-Supplemental Figure 1: High-resolution imaging of somite-like structures in day 10245 organoids

1025 Figure 3-Figure Supplement 2: Additional qPCR data and inter-organoid phenotypic 1026 variance of secondary somite phenotype screen in human PM organoids

1027 Figure 3-Supplemental Figure 3: The optimized Somitoid differentiation protocol is 1028 reproducible across experiments and different cell lines

1029 Figure 4-Figure Supplement 1: Single-cell RNA sequencing analysis of differentiating 1030 human PM organoids, NMp marker genes

1031 Figure 4-Figure Supplement 2: Single-cell RNA sequencing analysis of differentiating 1032 human PM organoids, PSM marker genes

1033 Figure 4-Figure Supplement 3: Single-cell RNA sequencing analysis of differentiating 1034 human PM organoids, Somite marker genes

1035 Figure 4-Figure Supplement 4: Single-cell RNA sequencing analysis of differentiating 1036 human PM organoids, cluster-based marker gene identification and HOX gene analysis 1037 Figure 4-Supplemental Figure 5: Single-cell RNA sequencing analysis of differentiating 1038 Somitoids reveals downregulation of WNT, FGF, and NOTCH target genes in day 5 1039 somite-like cells

1040 Figure 5-Supplemental Figure 1: Differentiation of Somitoid-derived cells towards 1041 skeletal muscle 


\section{Supplemental Files}

1043 Supplemental file 1: Supplemental Table 1, cluster-based marker genes of scRNA-seq 1044 dataset

1045 Supplemental file 2: Supplemental Table 2, RT-qPCR primer sequences

1046 Figure 3-Video 1, confocal z-stacks of Somitoids and control organoids immunostained 1047 for PAX3 and NCAD showing in vitro somite-like structures

\section{Source Data Files}

1049 Fig 1-Source Data 1: qPCR raw data of PM organoid differentiation.

1050 Fig 2-Source Data 1: Quantification of organoid diameter from primary screen.

1051 Fig 2-Source Data 2: Quantification of average PAX3 levels per organoid from primary 1052 screen.

1053 Fig 3-Source Data 1: Quantification of average PAX3 levels per organoid from 1054 secondary screen.

1055 Fig 3-Source Data 2: Quantification of somite-like structures of secondary screen.

1056 Fig 5-Source Data 1: Comparative somite size quantification of in vitro somite-like 1057 structures and human somites from the Carnegie collection.

1058 Fig 5-Source Data 2: qPCR raw data of sclerotome differentiation.

1059 Fig 3-Supplemental Fig 2-Source Data 1: qPCR data of calculated expression levels of 1060 selected treatment regimes from secondary screen.

1061 Fig 3-Supplemental Fig 3-Source Data 1: Quantification of somite-like structures of 1062 technical replicates using the NCRM1 cell line.

1063 Fig 3-Supplemental Fig 3-Source Data 2: Quantification of somite-like structures of 1064 biological replicates using the NCRM1, ACTB-GFP, and WTC cell lines. 
Fig 1, Budjan et al.

A

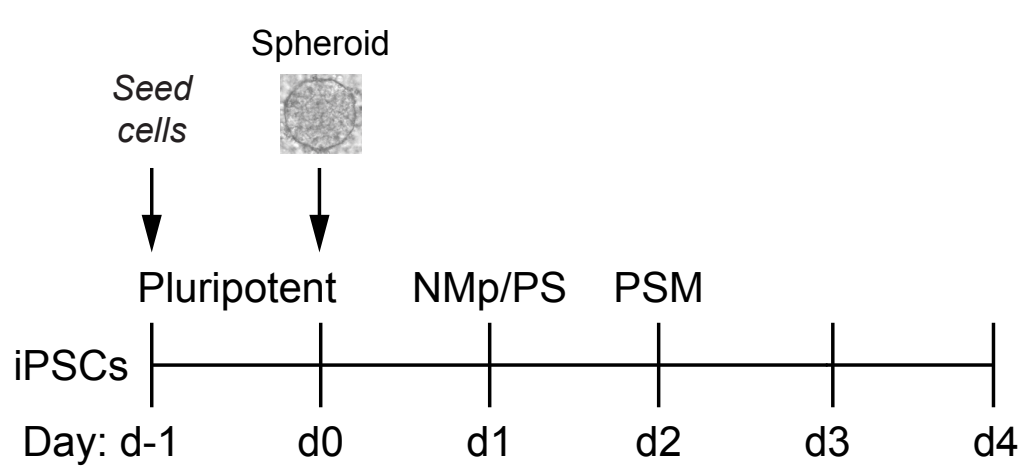

Aggregation

mTESRROCK

CHIR/LDN (CL)

$\mathrm{CL} / \mathrm{FGF}$

B

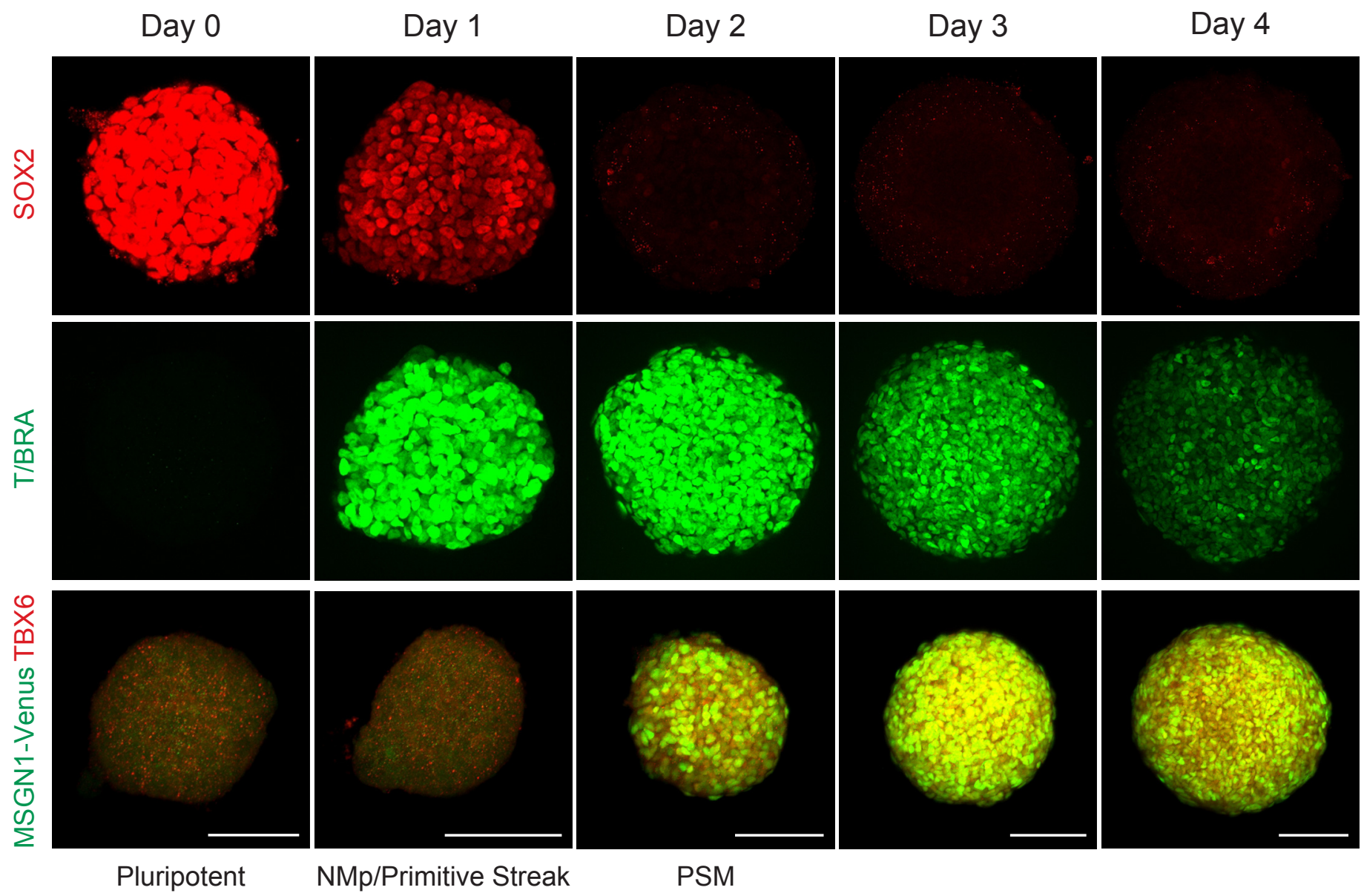

C

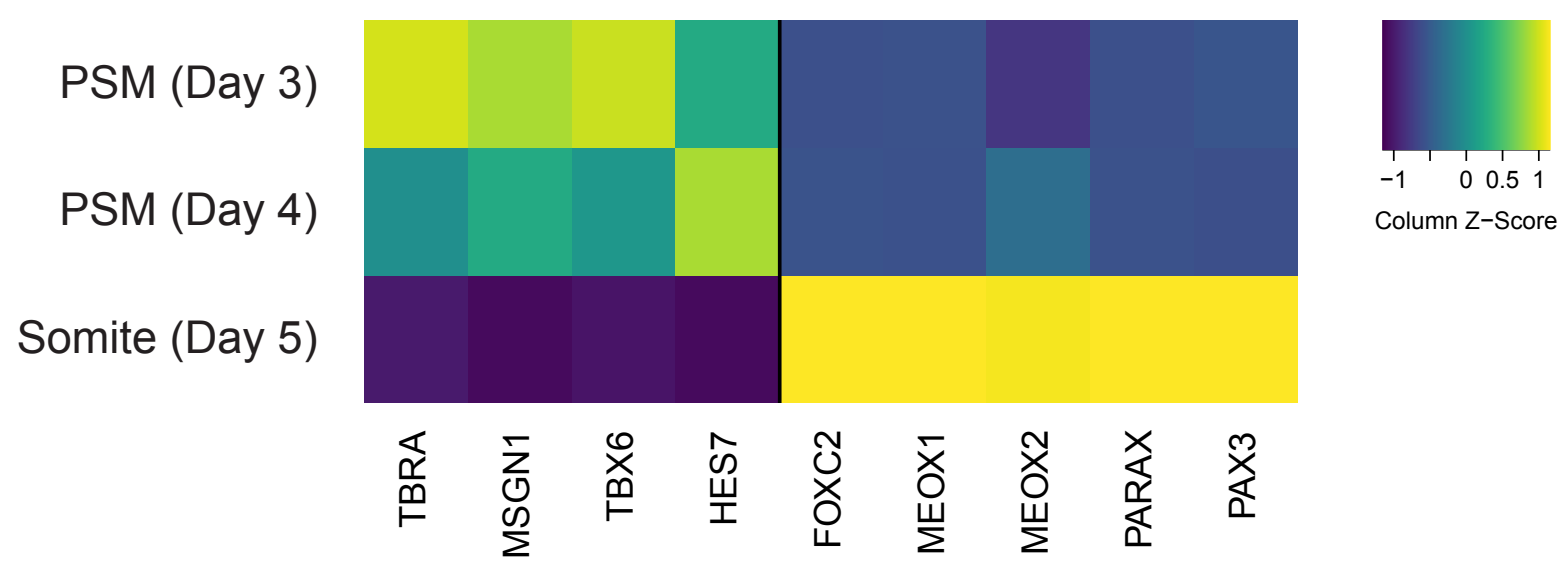


Fig 2, Budjan et al.

A

B
Combinatorial Pilot Screen for Somite Phenotype

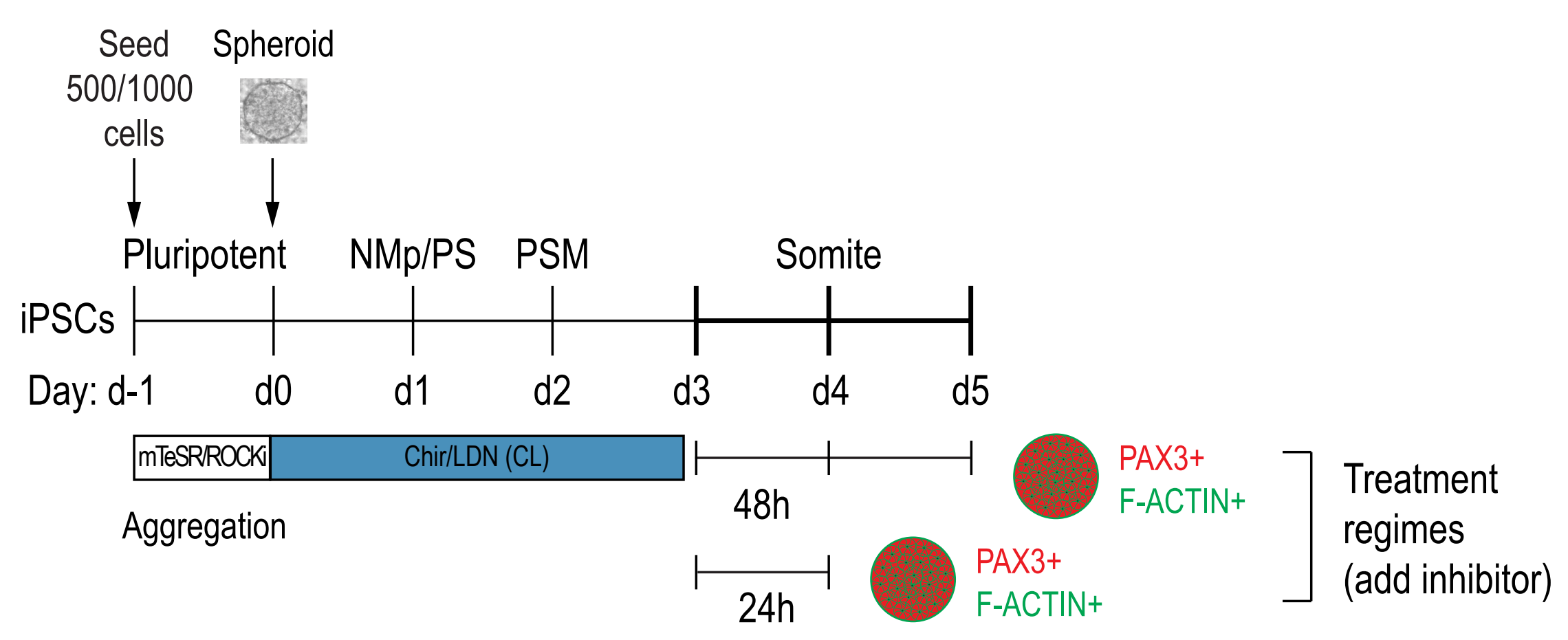

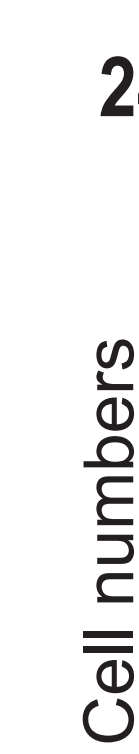

24

48 hours (Day 5)
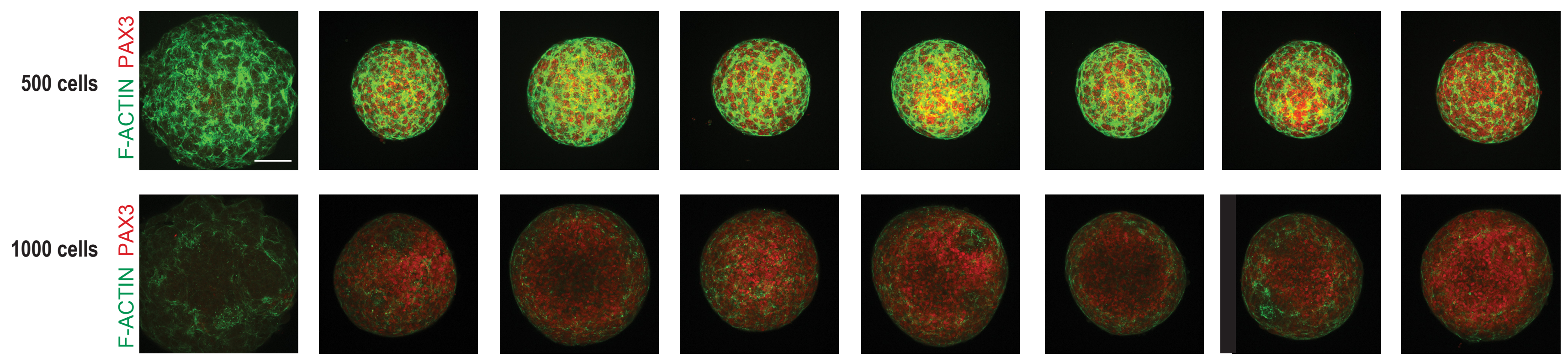

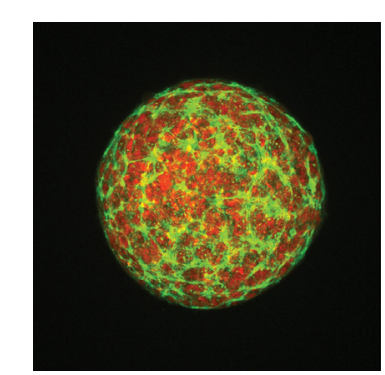

C

Somitoid Diameter quantification
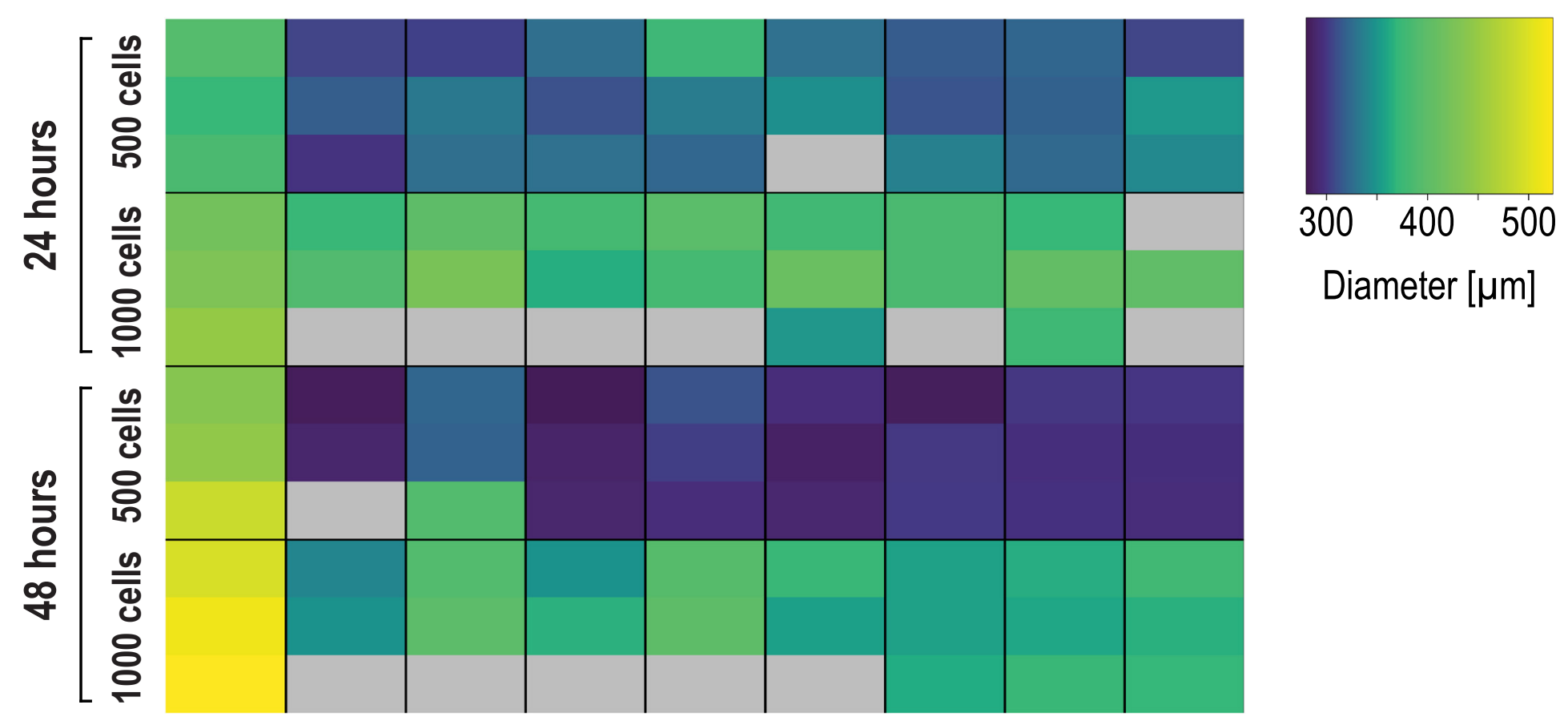

D

Somitoid Pax3 quantification
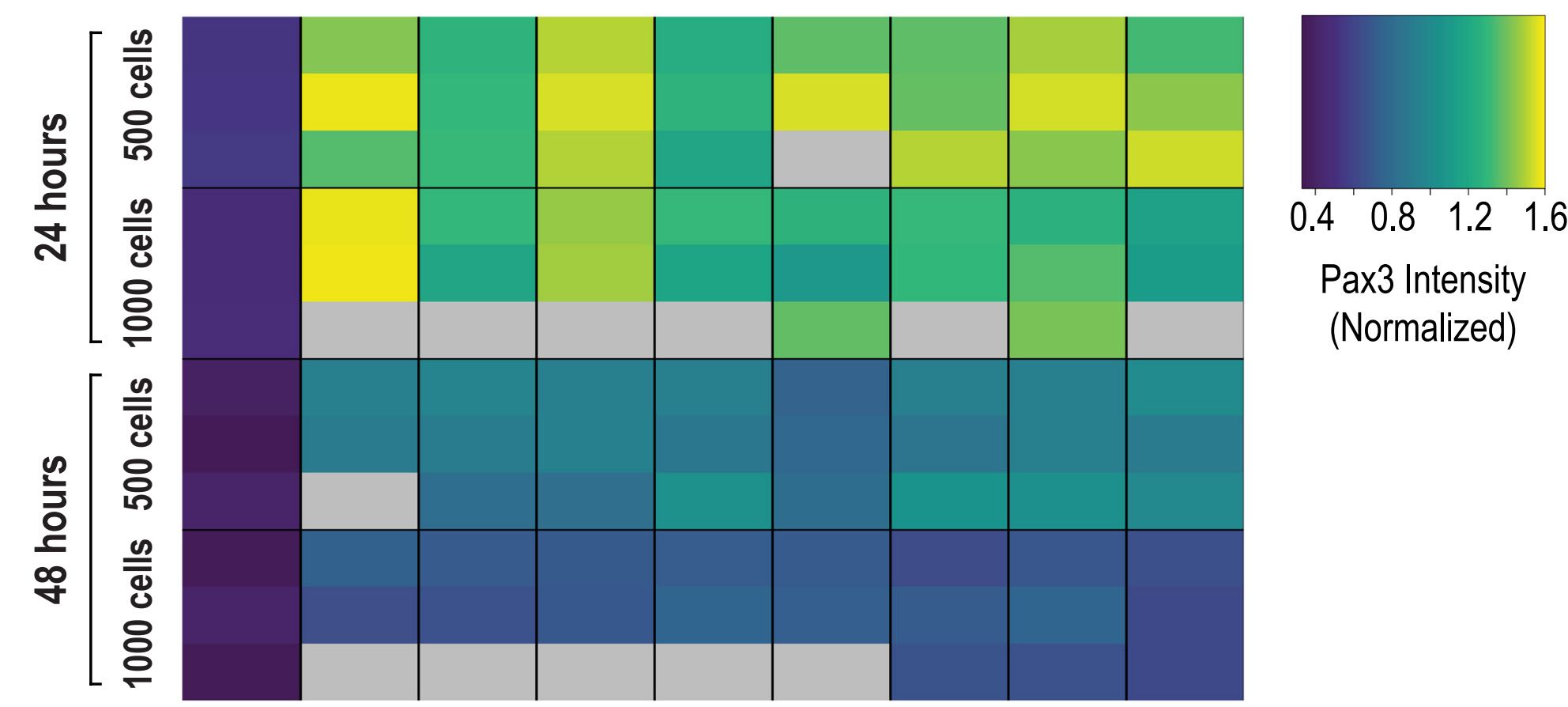

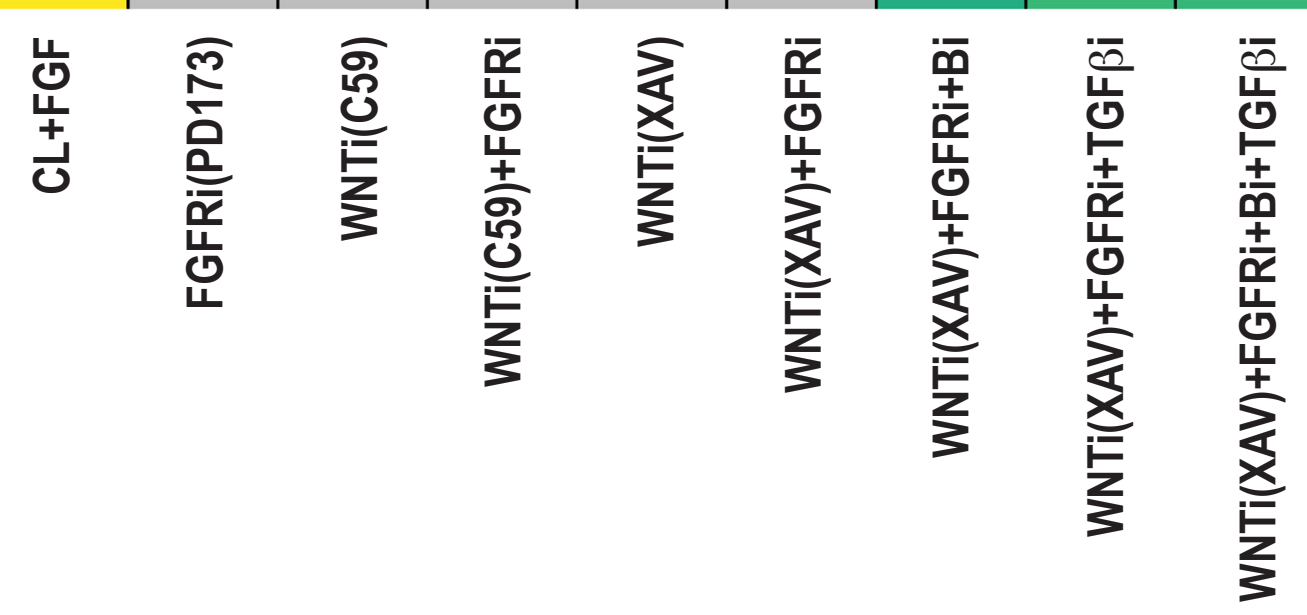

WNTi(XAV) FGFRi+TGF $\beta i+B i$
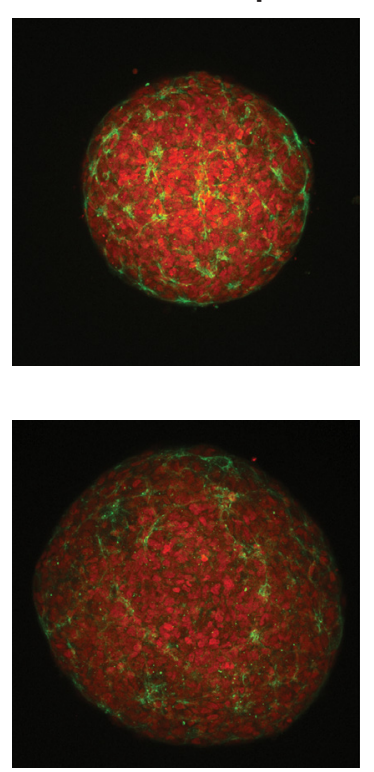

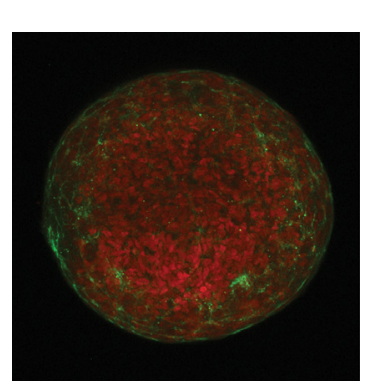


Fig 3, Budjan et al.

Combinatorial Screen for Somite Phenotype (Secondary)

A

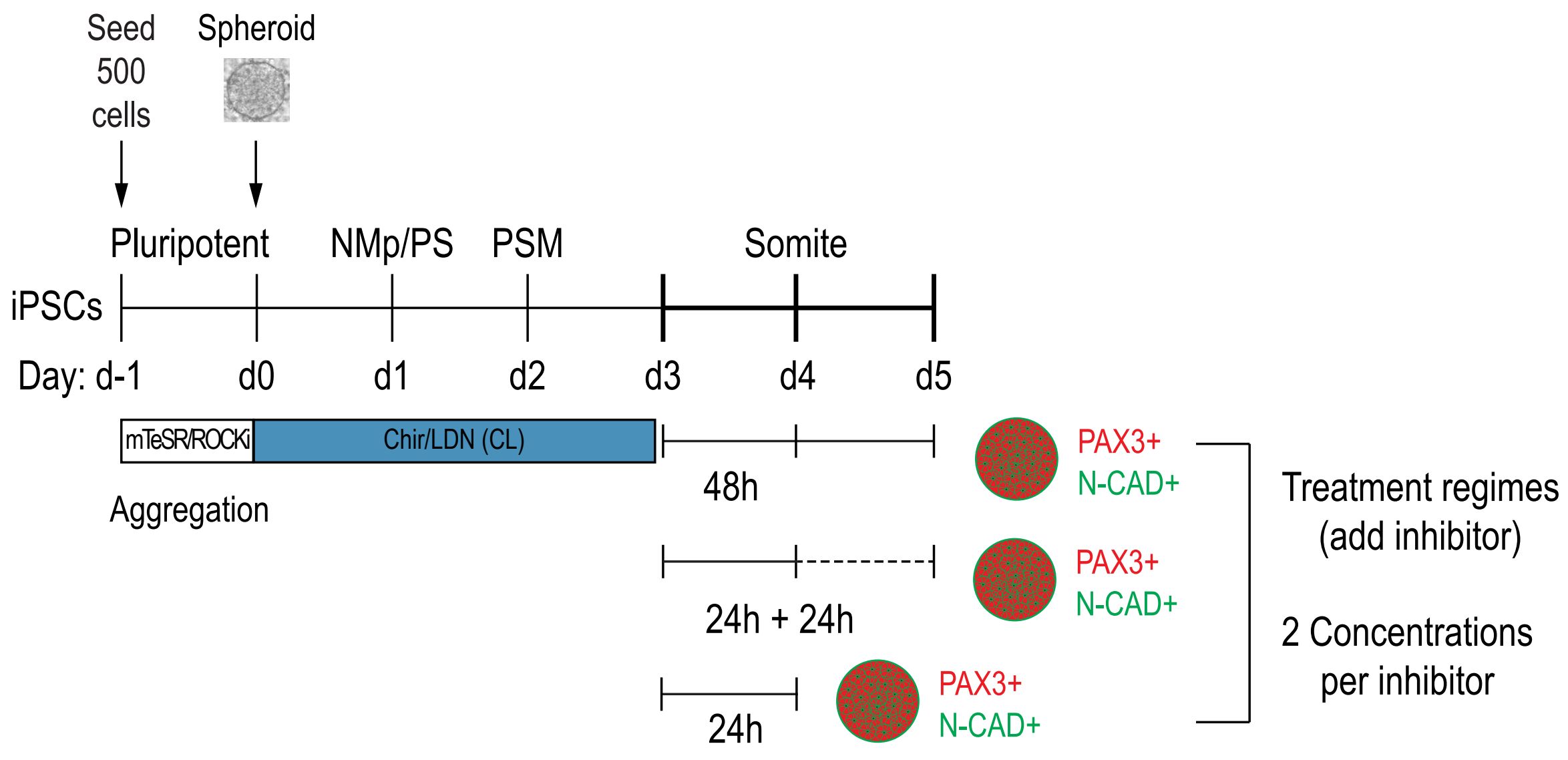

B

Somite quantification

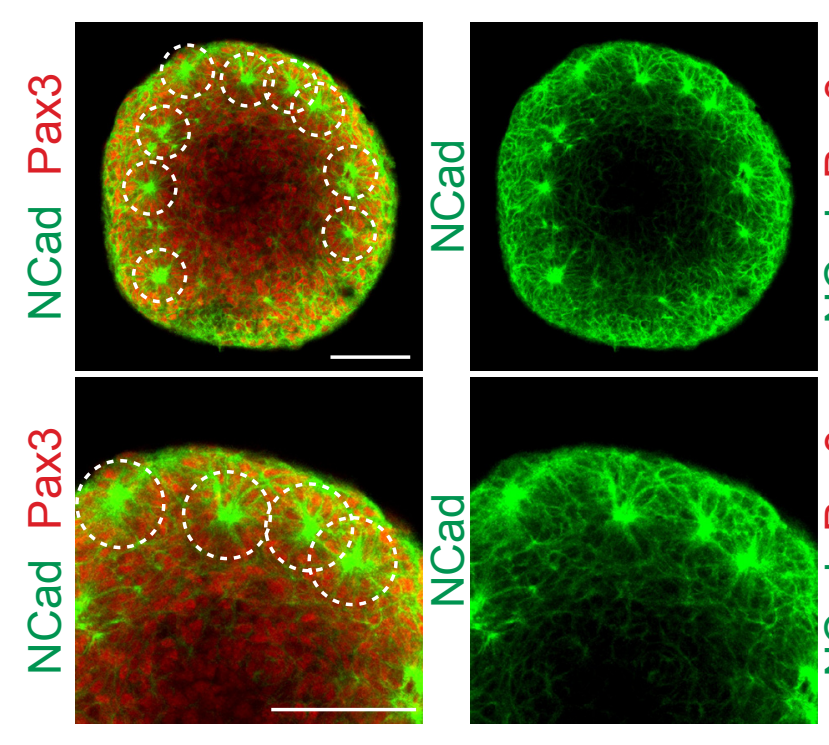

single z-section

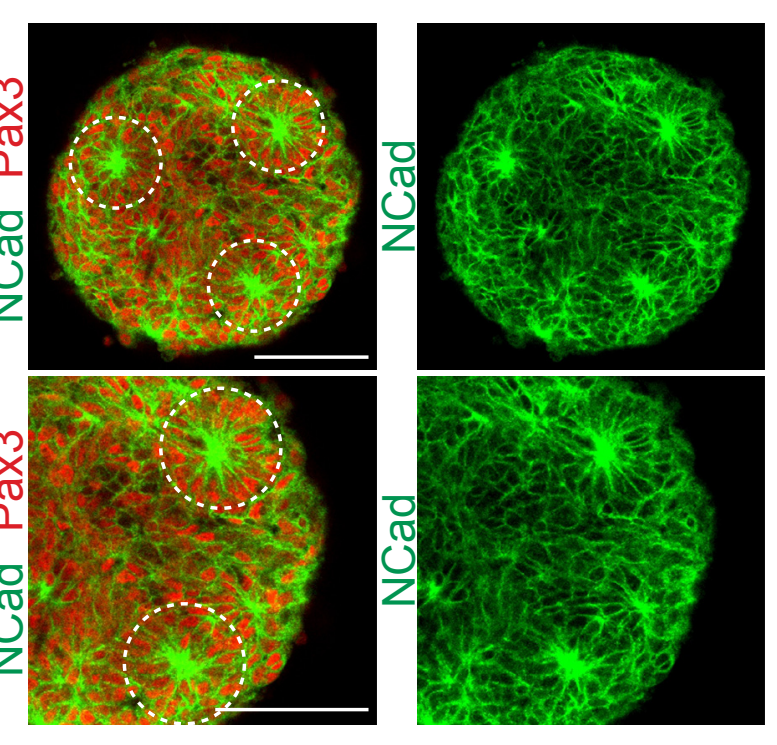

single z-section

C 24 hours (Day 4)
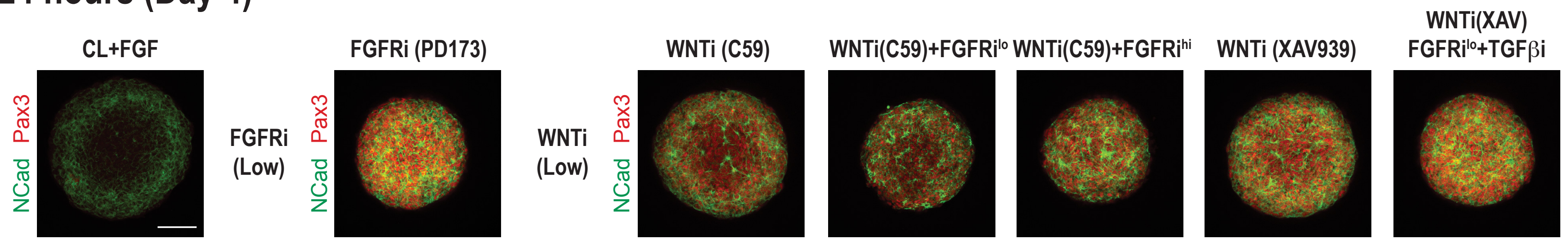

WNTi(XAV)
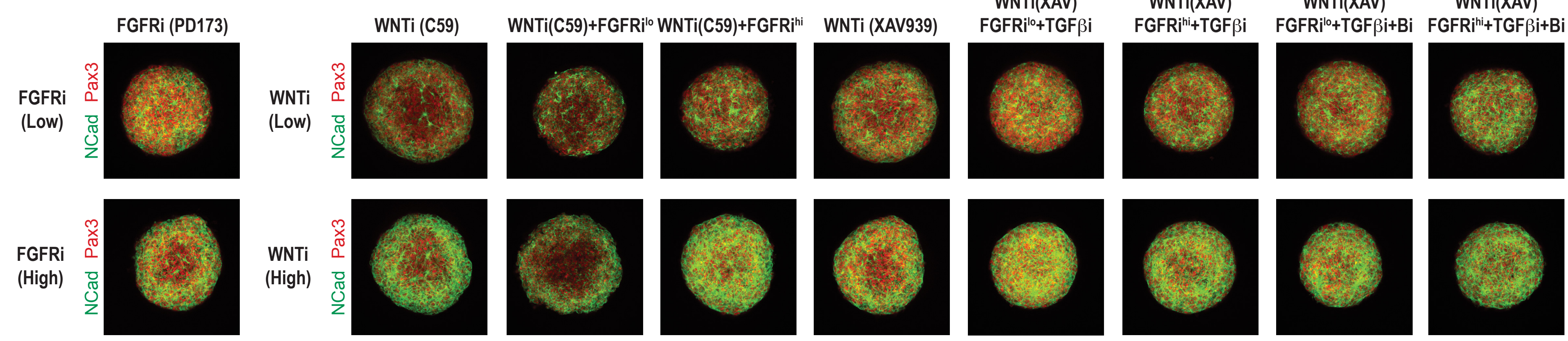

48 hours (Day 5)
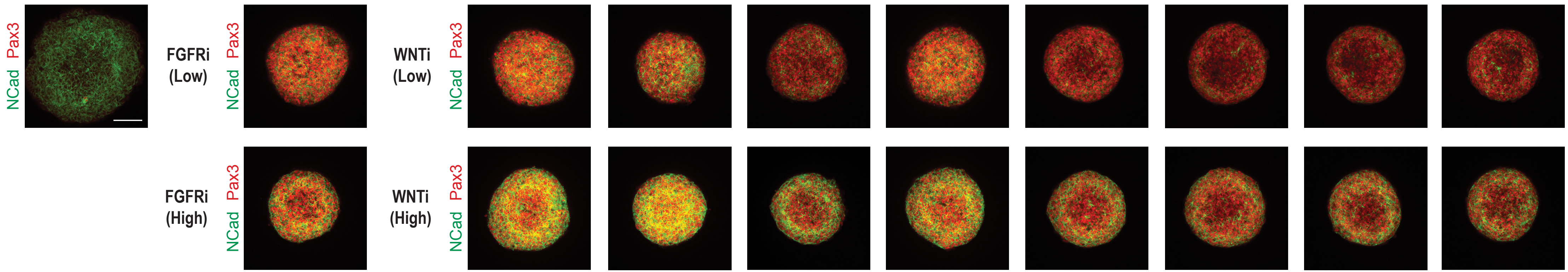

24h+24h (Day 5)
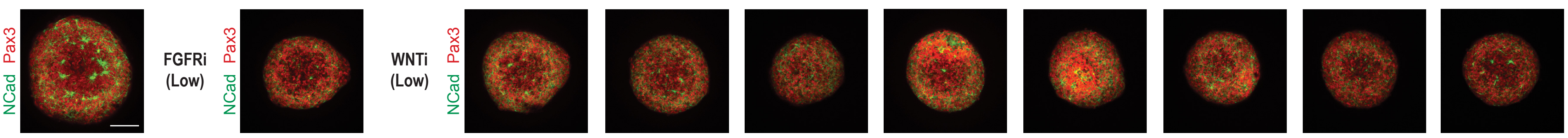

FGFR
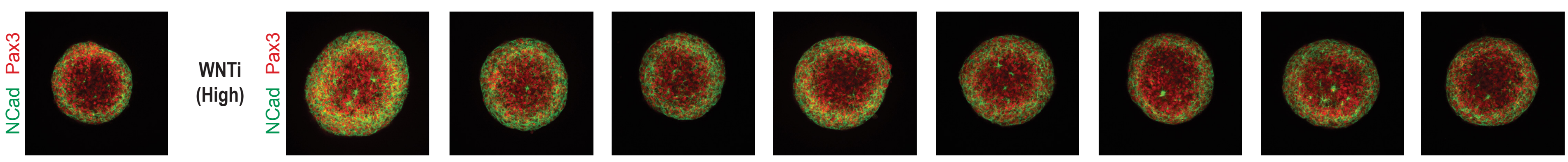

D

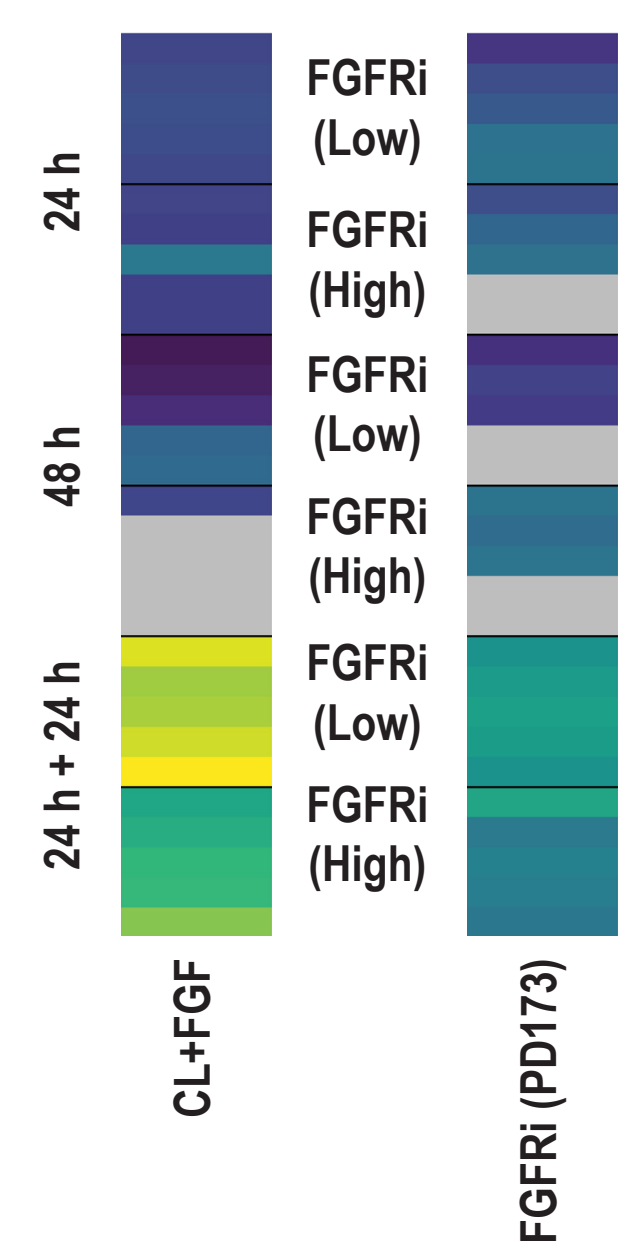

\section{PAX3 quantification}

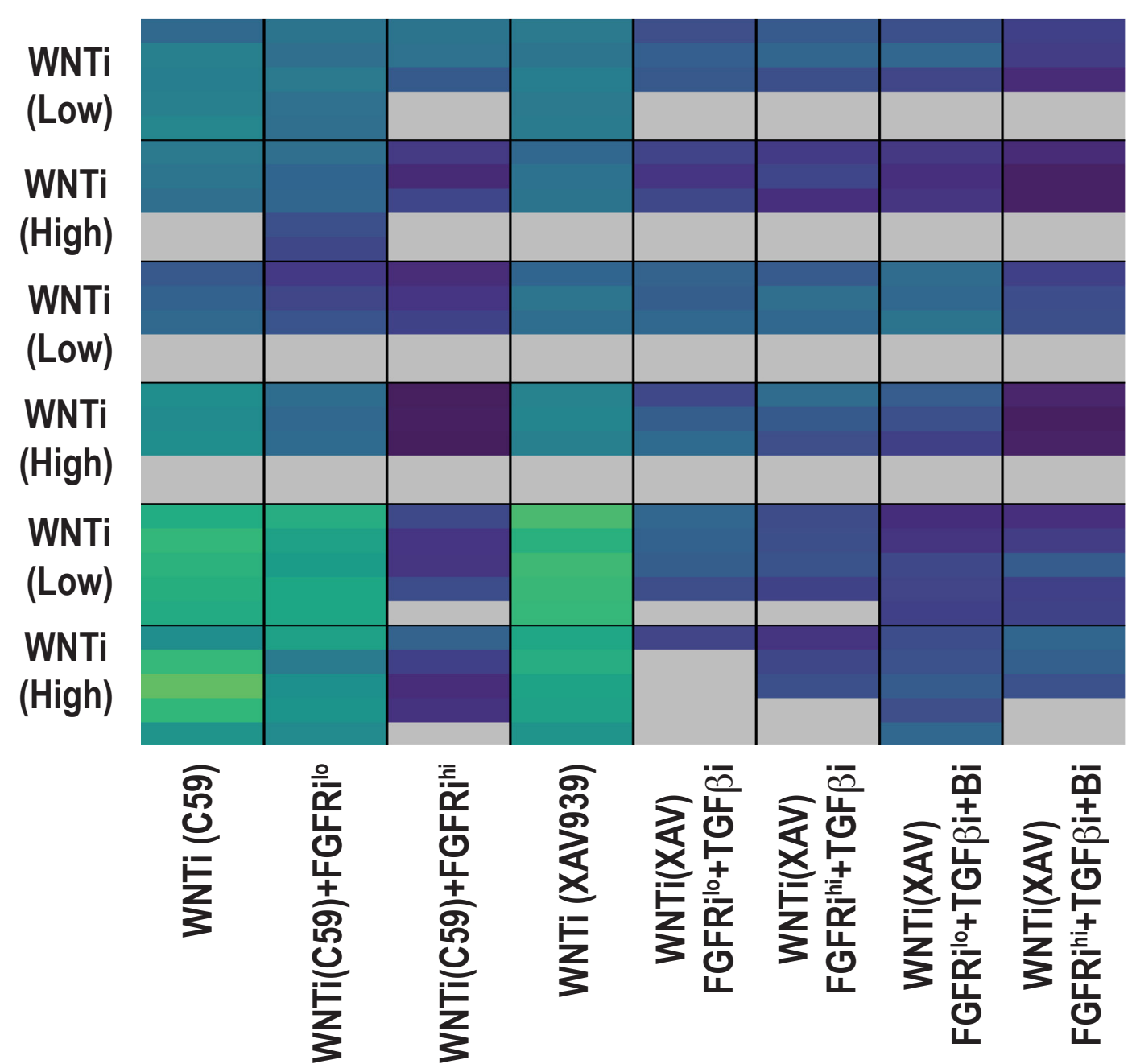

E
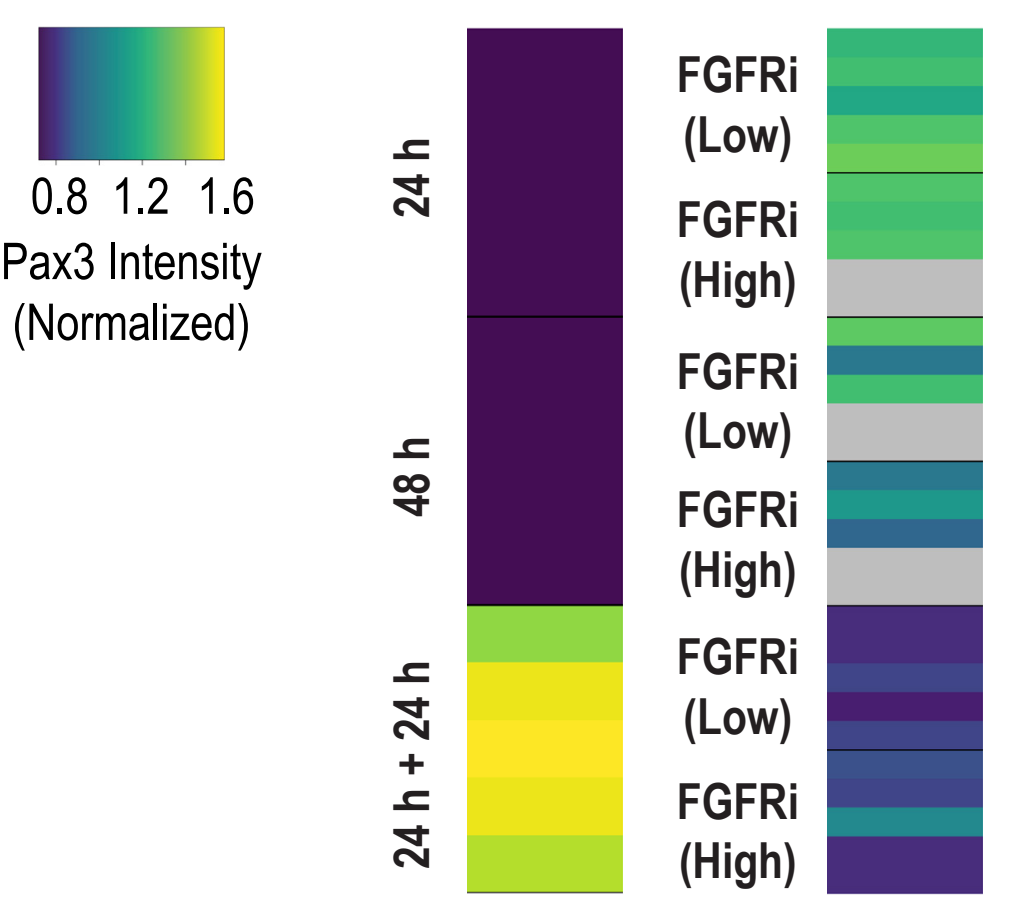

嵌

\section{Somite quantification}

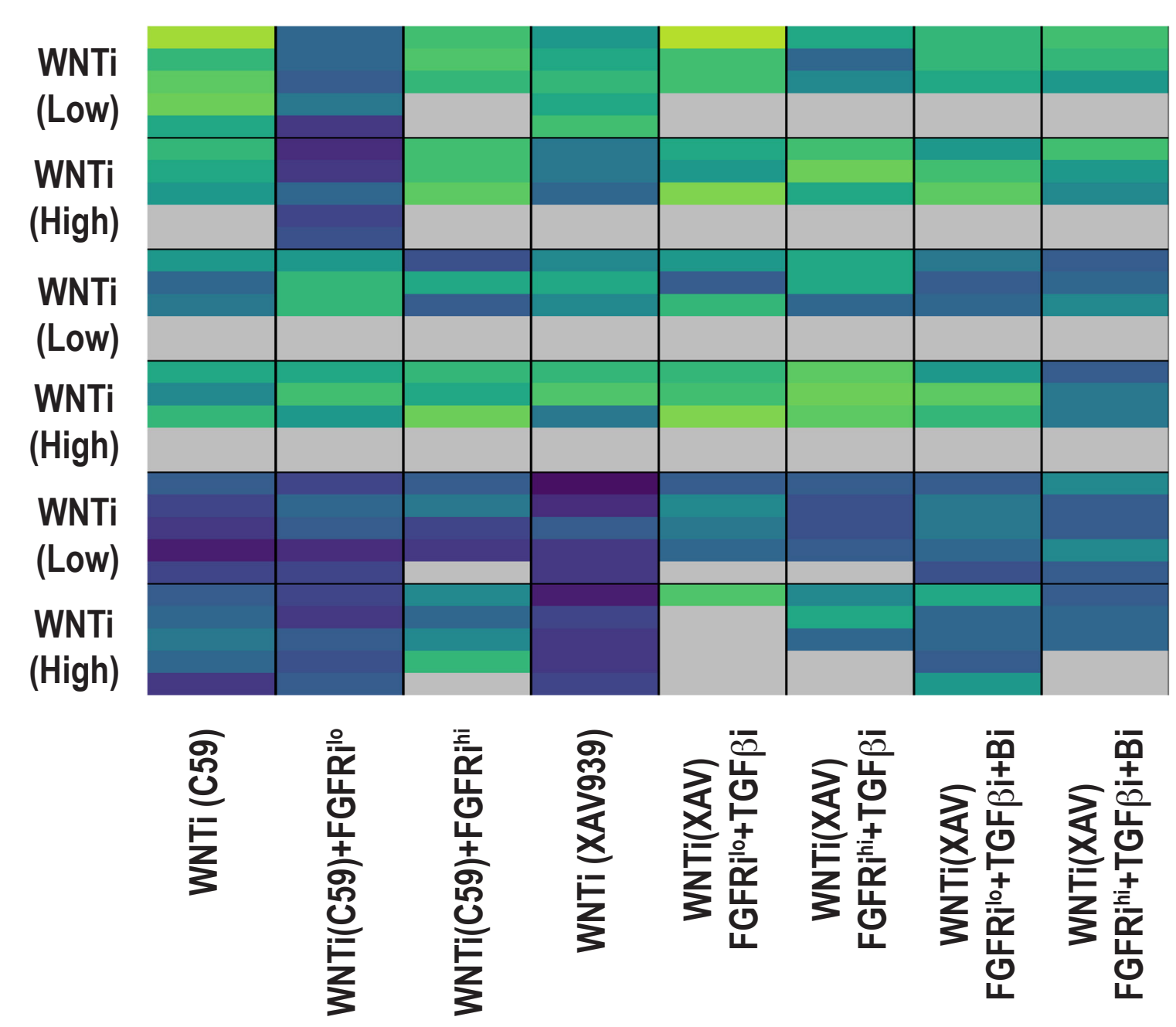




\section{Fig 4, Budjan et al.}

A

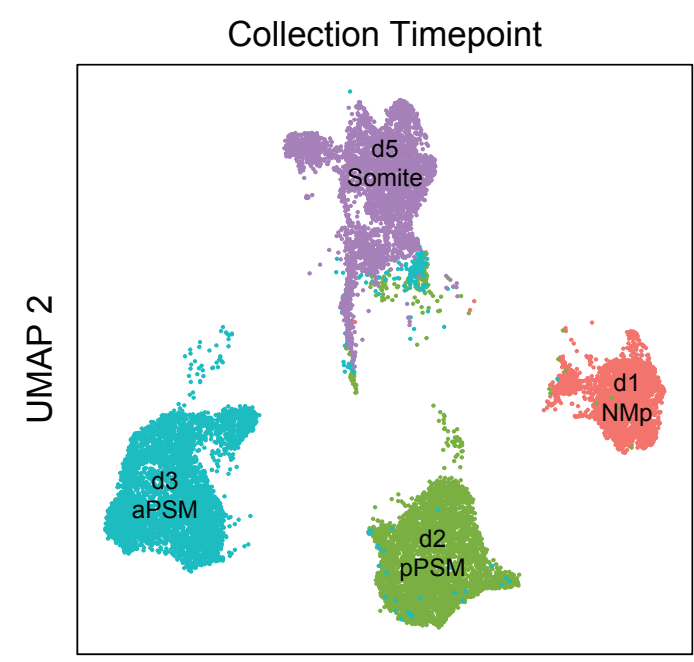

UMAP 1

C

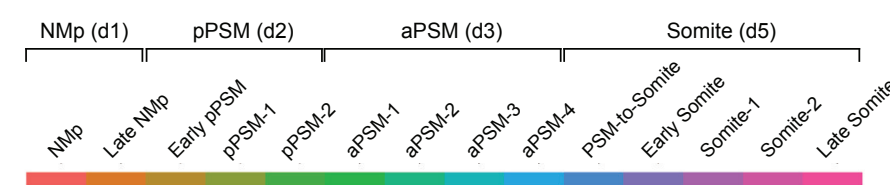

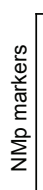

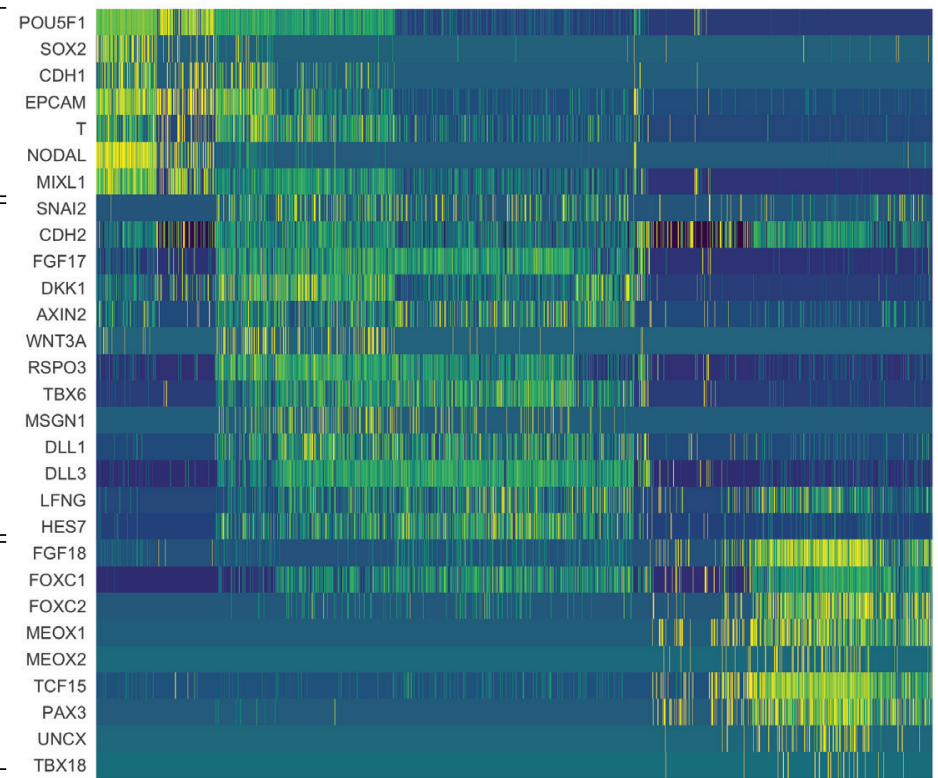

E

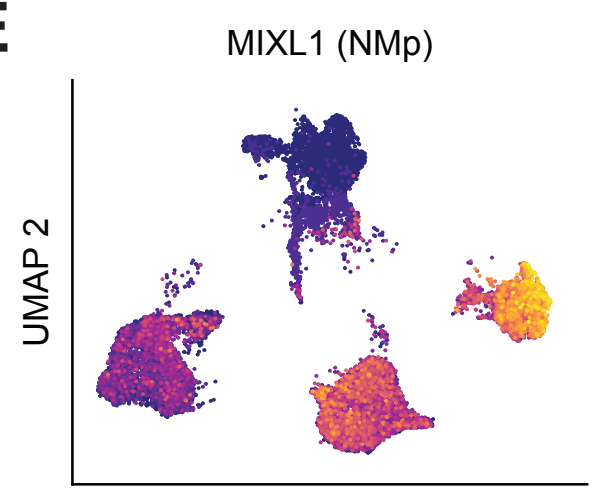

UMAP 1

B

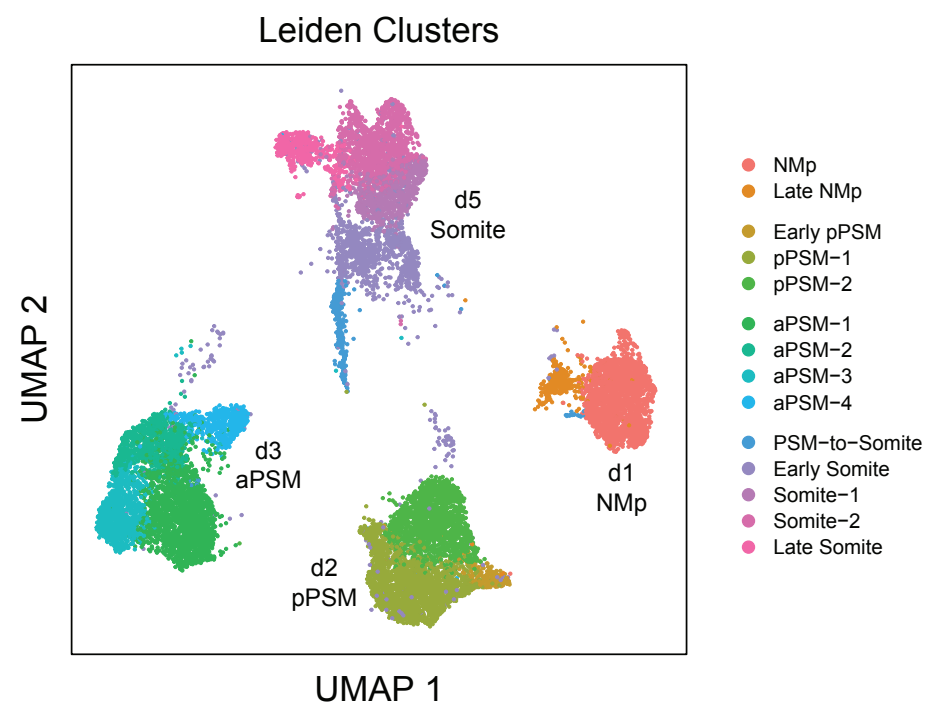

D

NMp (d1) $\Gamma_{\|} \quad \operatorname{pPSM}(\mathrm{d} 2) \quad$ aPSM (d3)

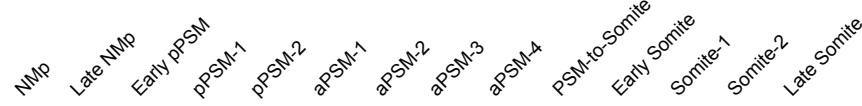

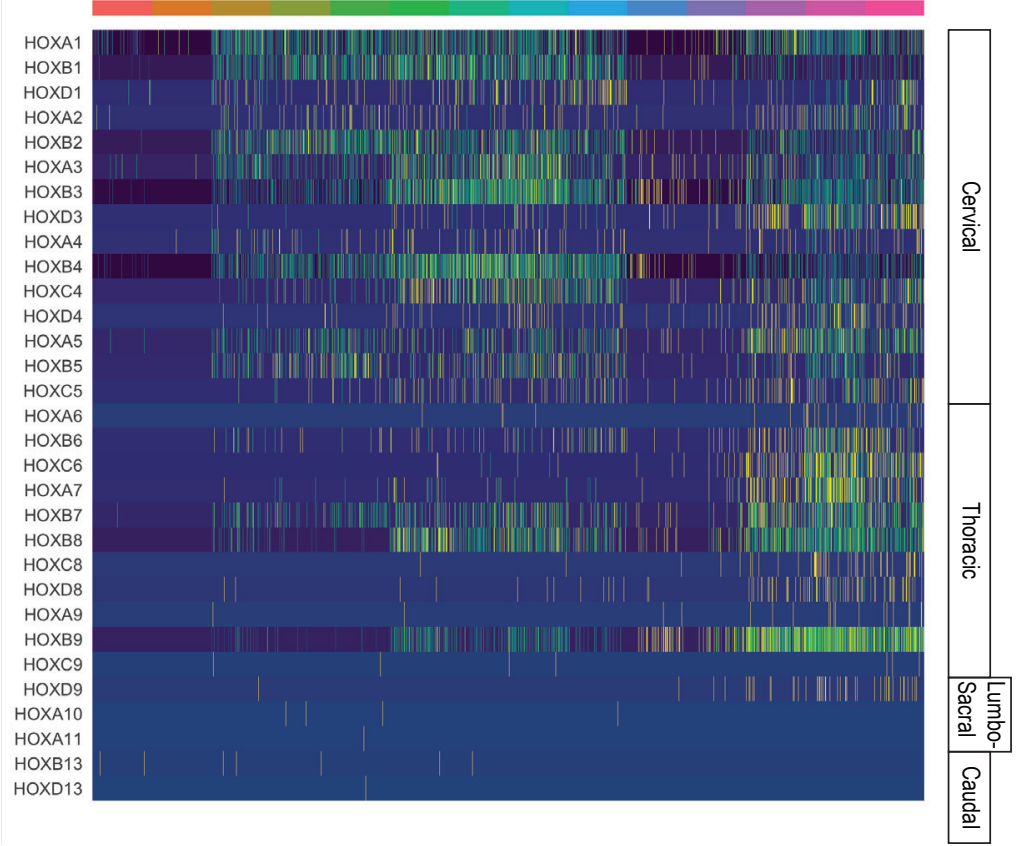

TCF15 (Somite)
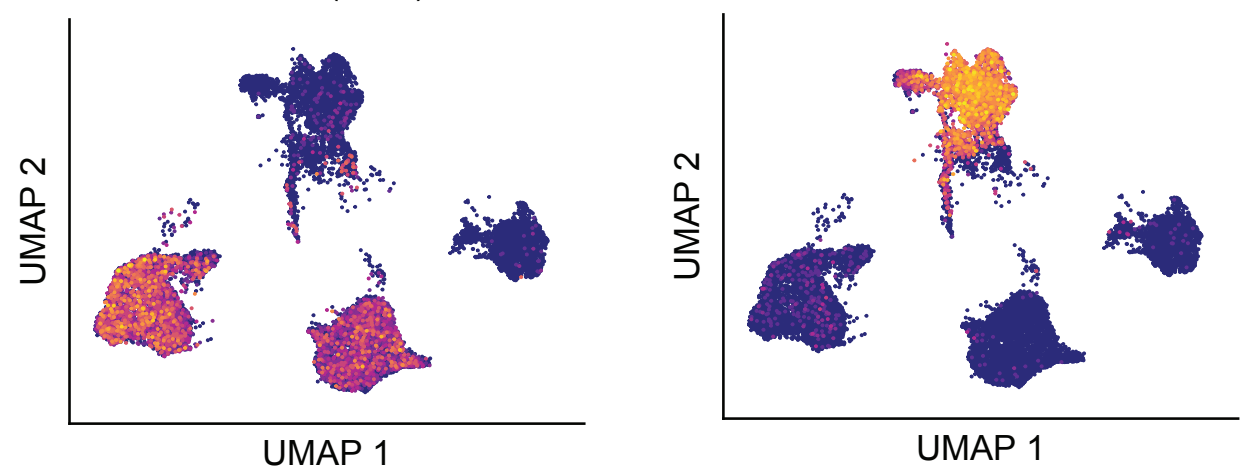
Fig 5, Budjan et al.

A

Day $5(C L+F G F, 24 h+24 h)$
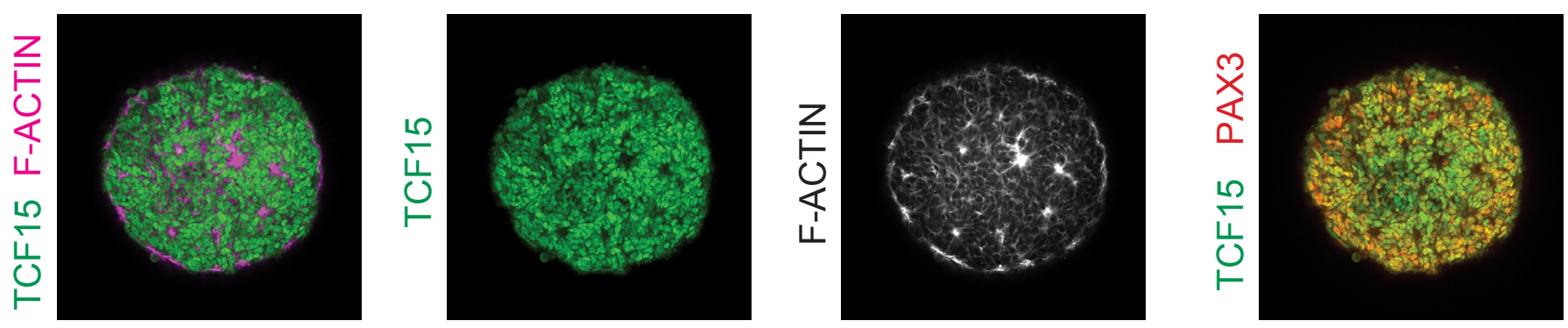

B

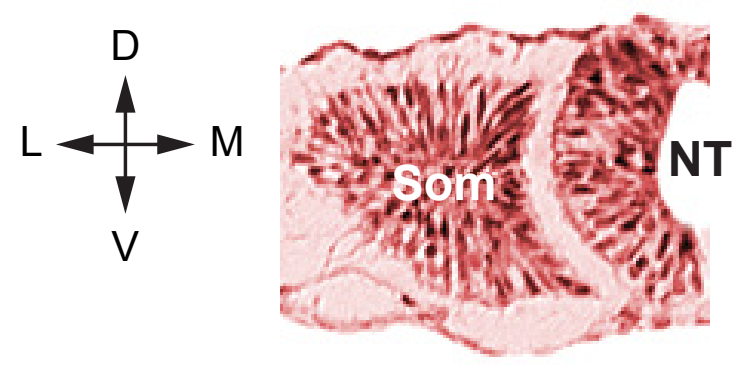

Carnegie Stage 10

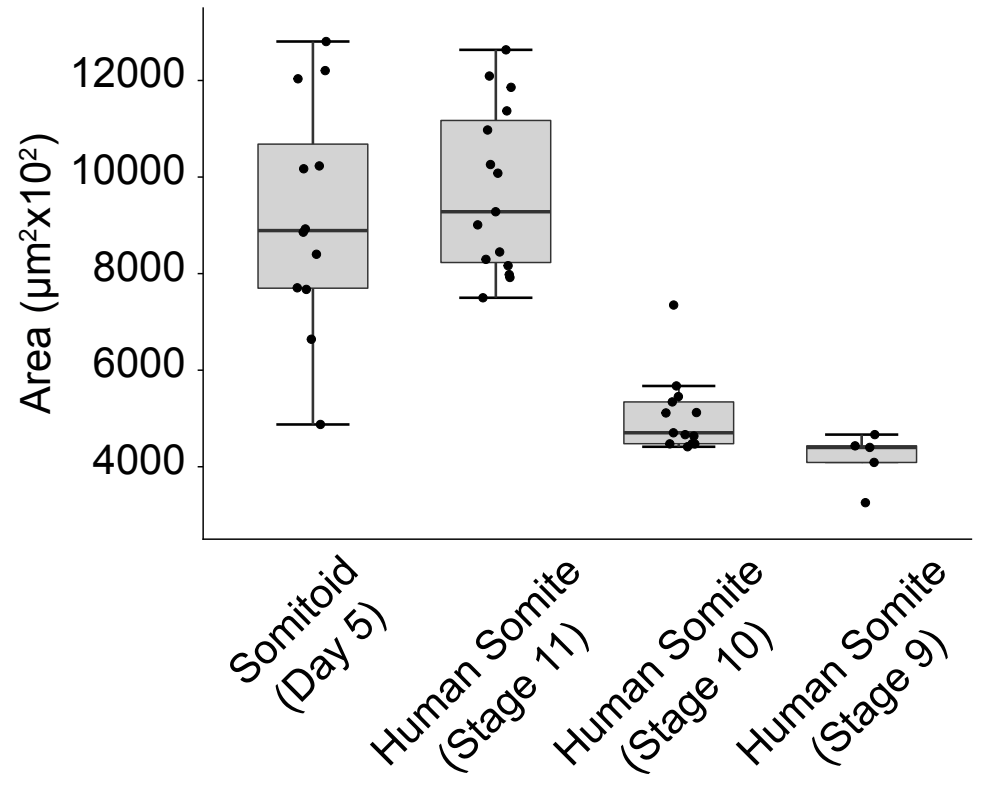

C

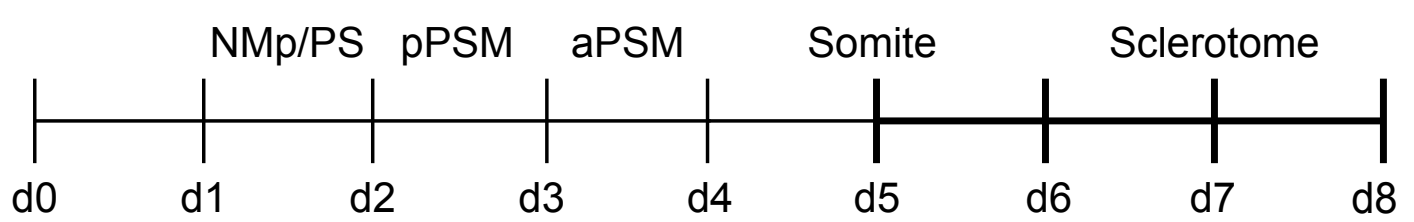

Somite (Day 5)

Sclerotome (Day 8)
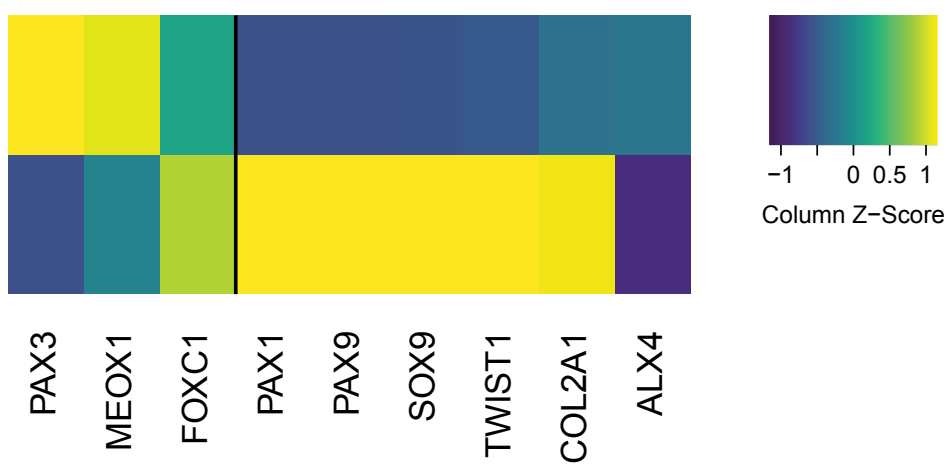

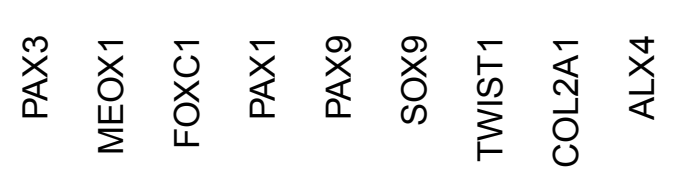

